\title{
Manifold mapping: a two-level optimization technique
}

\author{
D. Echeverría · P. W. Hemker
}

Received: 11 September 2007 / Accepted: 10 January 2008 / Published online: 26 March 2008

(c) Springer-Verlag 2008

\begin{abstract}
In this paper, we analyze in some detail the manifold-mapping optimization technique introduced recently [Echeverría and Hemker in space mapping and defect correction. Comput Methods Appl Math 5(2): 107--136, 2005]. Manifold mapping aims at accelerating optimal design procedures that otherwise require many evaluations of time-expensive cost functions. We give a proof of convergence for the manifold-mapping iteration. By means of two simple optimization problems we illustrate the convergence results derived. Finally, the performances of several variants of the method are compared for some design problems from electromagnetics.
\end{abstract}

Keywords Two-level optimization .

Nonlinear optimization - Surrogate optimization .

Simulation-based optimization $\cdot$ Space mapping

Mathematics Subject Classification (2000) 90C30 . $65 \mathrm{~K} 05 \cdot 49 \mathrm{M} 99 \cdot 49 \mathrm{Q} 99$

Dedicated to Wolfgang Hackbusch on the occasion of his 60th birthday.

Communicated by G. Wittum.

This research was supported by the Dutch Ministry of Economic Affairs through the project IOP-EMVT-02201 B.

D. Echeverría

Stanford University, Green Earth Sciences Bldg., 367 Panama Street, Stanford, CA 94305-2220, USA

e-mail: d.echeverria@stanford.edu

P. W. Hemker ( $\varangle)$

Centrum voor Wiskunde en Informatica (CWI),

Kruislaan 413, 1098 SJ, Amsterdam, The Netherlands

e-mail: p.w.hemker@cwi.nl

\section{Introduction}

Optimization problems in practice often require function evaluations that are very expensive to compute, and in most situations gradient information is not available. This is the case, e.g., for optimal design problems where the design simulations are based on complex finite element computations. As a consequence, many of the optimization processes require inconvenient long computing times.

In engineering, surrogate models have been used since long in analysis and design $[9,13,33]$ and recently they have been successfully applied to solving very time-consuming optimization problems $[1,12,18,23]$. In surrogate optimization the quality of the initial approximation is iteratively improved. A first surrogate yields the first iterate and with the use of it, the surrogate is improved. This iterative procedure is repeated until some stopping criteria are met.

The nature of the surrogates is problem dependent. We can clearly distinguish two different types of surrogates. If no a priori information is available, approximations can be obtained from scratch, in most cases combining experiment design strategies (e.g., Latin hypercube sampling [35] or orthogonal arrays [30]) with interpolation/approximation techniques (e.g., low degree polynomials [17], kriging [28] or radial basis functions [15]). The second type of approximations is found in situations where, because of, e.g., experience or simple rules of thumb, the derivation of the surrogate is simplified. An example of this is the use of lumped parameter models (e.g., magnetic [20], electric [6] or thermal [31] equivalent circuits).

Space mapping $[4,6]$ is a well-known surrogate-based optimization technique. Though it can be used in combination with any type of surrogate, it is traditionally applied with approximations of the second type described above. The space-mapping technique has been reported as an 
efficient minimization procedure in a large number of cases [6].

There are many variants of the space-mapping algorithm: aggressive space mapping (ASM) [5], trust-region aggressive space mapping (TRASM) [2], neural space mapping (NSM) [3] and implicit space mapping (ISM) [7] being the most significant examples. Although all these schemes do not always converge to the right solution [20], the solution obtained is generally acceptable for practical purposes. Recently, in [8] the original space-mapping approach is modified according to the framework proposed in [1]. At the expense of incorporating exact gradient information, the new scheme yields convergence to the accurate optimum.

Defect-correction theory [10] helps in understanding the space-mapping concept. Defect correction is the underlying basis of a great number of mathematical techniques that essentially solve a complex problem by the iterative use of a simpler one (e.g., Newton's method, relaxation procedures [11], iterative refinement [11] or multigrid methods [26]). Though defect-correction theory was originally developed for linear or nonlinear systems of equations, it can also be applied in optimization problems. Space-mapping procedures can be seen as special cases of defect-correction iteration [20] and this can be used to explain in which case the space-mapping approach does or does not fail to find the correct solution.

Manifold mapping [20] is an alternative surrogate-based optimization technique. Manifold mapping can be used without computing exact gradient information and it has provable convergence to the right solution. This paper presents a thorough analysis of the manifold-mapping (MM) approach and it is structured as follows. In Sect. 2 the basic terminology and some general assumptions are introduced. The manifold-mapping approach, together with the algorithms derived, are described in Sect. 3. In this section it is also shown that, under mild assumptions, the fixed point for all MM algorithms/procedures is the accurate optimum. Convergence theorems for all these schemes are given in Sect. 4 and they are illustrated by means of two simple design problems in Sect. 5. Finally, in this section the manifold-mapping technique is compared with other efficient methods for optimal design problems from the field of electromagnetics.

\section{Problem statement}

The optimization problem Let the specification of the aim (the data) in an optimization problem be denoted by $\mathbf{y} \in Y \subset$ $\mathbb{R}^{m}$. Since the true mechanism how this aim can be achieved can be extremely complex, or even impossible to describe in all its details, we study it by mathematical models. Often, such models appear in several degrees of sophistication. It is the purpose of manifold mapping to exploit the simpler models by combining their efficiency in computation with the accuracy of the more complex ones. Thus, we distinguish two types of model: fine and coarse.

The fine model The fine model response function is denoted by $\mathbf{f}: X \subset \mathbb{R}^{n} \rightarrow \mathbb{R}^{m}$, and $\mathbf{x} \in X$ is the control variable. The set $X$ of possible control variables is usually a closed and bounded subset of $\mathbb{R}^{n}$. The fine model is assumed to be accurate but expensive to evaluate. We assume that $\mathbf{f}(\mathbf{x})$ is differentiable but its Jacobian matrix $J_{\mathbf{f}}(\mathbf{x})=\mathbf{d f} / \mathbf{d x}$ is generally supposed to be unavailable.

For the optimization problem a fine model cost function, $F(\mathbf{x})=\|\mathbf{f}(\mathbf{x})-\mathbf{y}\|$ is defined, which is a measure for the discrepancy between the aim and a particular response of the mathematical model. This cost function should be minimized. So we look for

$\mathbf{x}_{\mathbf{f}}^{*}=\underset{\mathbf{x} \in X}{\operatorname{argmin}}\|\mathbf{f}(\mathbf{x})-\mathbf{y}\|$.

For simplicity, in this paper we take for $\|\cdot\|$ the Euclidean norm on $\mathbb{R}^{m}$, denoted by $\|\cdot\|$. Not every optimization problem is of this type, but many practical design situations can finally be reduced to this model-specification structure.

A design is called reachable if there exists an $\mathbf{x}_{\mathbf{f}}^{*} \in X$ such that $\mathbf{f}\left(\mathbf{x}_{\mathbf{f}}^{*}\right)=\mathbf{y}$. This situation can often be expected when $n \geq m$ since in that situation the number of degrees of freedom in the design is larger than or equal to the number of specifications. Reachable designs can be formulated as equations and, hence, they can generally be solved as nonlinear systems. The original defect-correction iteration [10] can be directly applied. For this reason, in this work we will particularly analyze the overdetermined case $n<m$. We formulate this as our first assumption.

Assumption 1 The dimension of the space of possible aims exceeds the dimension of the control space: $n<m$.

The coarse model The coarse model is denoted by $\mathbf{c}: Z \subset$ $\mathbb{R}^{n} \rightarrow \mathbb{R}^{m}$ with $\mathbf{z} \in Z$ the coarse model control variable. In this work, for simplicity, we consider $X=Z$, but the general case can be dealt with by the introduction of an additional mapping $\overline{\mathbf{p}}: X \rightarrow Z$, as used in [20]. In contrast to the fine model, the coarse model is assumed to be cheap to evaluate but less accurate. For the coarse model we have the coarse model cost function, $C(\mathbf{z})=\|\mathbf{c}(\mathbf{z})-\mathbf{y}\|$. We denote its minimizer by $\mathbf{x}_{\mathbf{c}}^{*} \in Z$,

$\mathbf{x}_{\mathbf{c}}^{*}=\underset{\mathbf{z} \in Z}{\operatorname{argmin}}\|\mathbf{c}(\mathbf{z})-\mathbf{y}\|$.

Also for this cost function we consider in this paper the Euclidean norm. The Jacobian matrix $J_{\mathbf{c}}(\mathbf{z})=\mathbf{d c} / \mathbf{d z}$ can be assumed to be available at no significant computational cost. 
Manifolds and constraints If the functions $\mathbf{f}(\mathbf{x})$ and $\mathbf{c}(\mathbf{x})$ are sufficiently smooth, the sets $\mathbf{f}(X) \subset \mathbb{R}^{m}$ and $\mathbf{c}(X) \subset \mathbb{R}^{m}$ can be considered as differential manifolds. In the appendix we summarize a number of manifold-related definitions (from [36]) relevant for our discussion. For simplicity and without loss of generality, we just consider one particular chart of the manifold that covers a sufficiently large neighborhood of the solution region. We thus make the following assumption.

Assumption 2 The sets $\mathbf{f}(X)$ and $\mathbf{c}(X)$ are differentiable manifolds of class $C^{2}$.

We need the concept of the tangent plane for a manifold at a point, which is used throughout this work.

Definition 1 Let $M$ be a differentiable manifold and $\mathbf{v} \in M$ a point in that manifold. The tangent plane for $M$ at $\mathbf{v} \in M$ is defined as the affine space spanned by the Jacobian of $\varphi$ at $\mathbf{x}$, being $\varphi$ any chart in the atlas of the manifold such that $\varphi(\mathbf{x})=\mathbf{v}$.

Since we only consider one chart we will denote the tangent plane for $\mathbf{f}(X)$ at $\mathbf{f}(\mathbf{x})$ simply by the Jacobian of $\mathbf{f}$ at $\mathbf{x}$, i.e., $J_{\mathbf{f}}(\mathbf{x})$. Thus the tangent plane is well defined. Similarly, $J_{\mathbf{c}}(\mathbf{x})$ will denote the tangent plane for $\mathbf{c}(X)$ at $\mathbf{c}(\mathbf{x})$.

We can state a general constrained optimization problem as follows

$$
\begin{aligned}
\mathbf{x}_{\mathbf{f}}^{*} & =\underset{\mathbf{x} \in X}{\operatorname{argmin}}\|\mathbf{f}(\mathbf{x})-\mathbf{y}\|, \\
X & =\left\{\mathbf{x} \in \mathbb{R}^{n} ; \mathbf{k}_{\mathbf{f}}(\mathbf{x})=0, \quad \overline{\mathbf{k}}_{\mathbf{f}}(\mathbf{x}) \geq 0\right\},
\end{aligned}
$$

where $\mathbf{f}: \mathbb{R}^{n} \rightarrow \mathbb{R}^{m}, \mathbf{k}_{\mathbf{f}}: \mathbb{R}^{n} \rightarrow \mathbb{R}^{n_{\mathbf{k}}}$ and $\overline{\mathbf{k}}_{\mathbf{f}}: \mathbb{R}^{n} \rightarrow \mathbb{R}^{\bar{n}_{\mathbf{k}}}$ are assumed to be differentiable, and $n_{\mathbf{k}}$ and $\bar{n}_{\mathbf{k}}$ are the number of equality and inequality constraints, respectively. In general we cannot expect the model and constraints to be defined over the entire $\mathbb{R}^{n}$. For example, it makes no sense in many cases to consider negative lengths. But it is common in practice that $\mathbf{f}$ and $\mathbf{k}_{\mathbf{f}}$ are correctly defined in $\widehat{X}$, the set where the inequality constraints are feasible

$\widehat{X}=\left\{\mathbf{x} \in \mathbb{R}^{n} ; \overline{\mathbf{k}}_{\mathbf{f}}(\mathbf{x}) \geq 0\right\}$.

It should be noted that box-constraints can be obtained with a proper choice for $\overline{\mathbf{k}}_{\mathbf{f}}(\mathbf{x})$. The inequality constraints do not generally reduce the dimensionality of the design space and therefore we prefer to rewrite (3) as

$\mathbf{x}_{\mathbf{f}}^{*}=\underset{\left\{\mathbf{x} \in \widehat{X} ; \mathbf{k}_{\mathbf{f}}(\mathbf{x})=0\right\}}{\operatorname{argmin}}\|\mathbf{f}(\mathbf{x})-\mathbf{y}\|$.

In order to have degrees of freedom left for optimization, the number of equality constraints $n_{\mathbf{k}}$ should be smaller than the number of design variables $n$, i.e., $n_{\mathbf{k}}<n$. We also assume that the set $X$ is a differentiable manifold in $\mathbb{R}^{n}$. We formalize this in the following assumption.

Assumption 3 The set $X$ is either a subset of $\mathbb{R}^{n}$ or a differentiable manifold in $\mathbb{R}^{n}$ of dimension $n-n_{\mathbf{k}}>0$.
Now we can write the constrained optimization problem (5) as (1), with $X$ a differentiable manifold.

In this work we only analyze those cases in which the functions $\overline{\mathbf{k}}_{\mathbf{f}}(\mathbf{x})$ and $\mathbf{k}_{\mathbf{f}}(\mathbf{x})$ are easy to compute (and thus it makes sense to take $Z=X$ ). Below in Remark 3, we indicate how more complex constraints can be handled.

If the equality constraints $\mathbf{k}_{\mathbf{f}}(\mathbf{x})$ can be evaluated easily and a chart $\varphi_{X}$ for $\bar{X}=\left\{\mathbf{x} \in \mathbb{R}^{n} ; \mathbf{k}_{\mathbf{f}}(\mathbf{x})=0\right\}$ in the region of interest can be obtained with not significant computational cost, then the constrained optimization problem can be restated with $\mathbf{f} \circ \varphi_{X}$ as the fine model and a subset of $\mathbb{R}^{n-n_{\mathbf{k}}}$ as the control space.

By the above argument we assume that the equality constraints can be eliminated and, hence, $X$ can be considered as a subset of $\mathbb{R}^{n}$, understanding $n$ as the number of design variables left after removing the equality constraints. As a consequence, both Jacobians $J_{\mathbf{f}}(\mathbf{x})$ and $J_{\mathbf{c}}(\mathbf{x})$ have rank $n$.

If $\mathbf{x}_{\mathbf{f}}^{*}$ is a local optimum of the constrained problem (3), then it satisfies the Karush-Kuhn-Tucker (KKT) conditions [29], i.e., there are two Lagrange multiplier vectors $\lambda_{\mathbf{f}}^{*} \in \mathbb{R}^{n_{\mathbf{k}}}$ and $\bar{\lambda}_{\mathbf{f}}^{*} \in \mathbb{R}^{\bar{n}_{\mathbf{k}}}$ such that

$$
\begin{aligned}
\mathbf{d} / \mathbf{d x}\left(F-\lambda_{\mathbf{f}}^{* T} \mathbf{k}_{\mathbf{f}}-\bar{\lambda}_{\mathbf{f}}^{* T} \overline{\mathbf{k}}_{\mathbf{f}}\right)\left(\mathbf{x}_{\mathbf{f}}^{*}\right) & =\mathbf{0}, \\
\mathbf{k}_{\mathbf{f}}\left(\mathbf{x}_{\mathbf{f}}^{*}\right) & =\mathbf{0}, \\
\overline{\mathbf{k}}_{\mathbf{f}}\left(\mathbf{x}_{\mathbf{f}}^{*}\right) & \geq \mathbf{0}, \\
\lambda_{\mathbf{f}}^{*}, \bar{\lambda}_{\mathbf{f}}^{*} & \geq \mathbf{0}, \\
\operatorname{diag}\left(\bar{\lambda}_{\mathbf{f}}^{*} \cdot \overline{\mathbf{k}}_{\mathbf{f}}\left(\mathbf{x}_{\mathbf{f}}^{*}\right)\right) & =\mathbf{0},
\end{aligned}
$$

where denotes here the vector direct product.

Remark 1 In the case of an unconstrained optimization based on the Euclidean norm, the KKT conditions represent the orthogonality between the tangent plane for $\mathbf{f}(X)$ at $\mathbf{x}_{\mathbf{f}}^{*}$ and the optimal model-specifications discrepancy $\mathbf{f}\left(\mathbf{x}_{\mathbf{f}}^{*}\right)-\mathbf{y}$, i.e., $J_{\mathbf{f}}^{T}\left(\mathbf{x}_{\mathbf{f}}^{*}\right)\left(\mathbf{f}\left(\mathbf{x}_{\mathbf{f}}^{*}\right)-\mathbf{y}\right)=0$.

The following concept will be very useful when formalizing the similarity between models.

Definition 2 We say that an optimization problem is locally convex at $\mathbf{x} \in \mathbb{R}^{n}$ if and only if there is a neighborhood $U_{\mathbf{x}}$ of $\mathbf{x}$ such that every point in $U_{\mathbf{x}}$ satisfying the KKT conditions is a local minimizer.

The assumption for a well-defined optimization problem In order to be sure that the problems we want to solve make sense, we have to assume that there exist solutions for these designs.

Assumption 4 The fine and coarse optimization problems, characterized by $\mathbf{y}, \mathbf{f}$ and $\mathbf{c}$, and $X$, are uniquely solvable, i.e.,

$\forall \mathbf{y} \in Y \exists ! \mathbf{x}_{\mathbf{f}}^{*} \in X \quad \mathbf{x}_{\mathbf{f}}^{*}(\mathbf{y})=\underset{\mathbf{x} \in X}{\operatorname{argmin}}\|\mathbf{f}(\mathbf{x})-\mathbf{y}\|$, 
and

$\forall \mathbf{y} \in Y \exists ! \mathbf{x}_{\mathbf{c}}^{*} \in X \quad \mathbf{x}_{\mathbf{c}}^{*}(\mathbf{y})=\underset{\mathbf{x} \in X}{\operatorname{argmin}}\|\mathbf{c}(\mathbf{x})-\mathbf{y}\|$.

In most practical cases this assumption is reasonable. If $X$ is a closed and bounded non-empty set and $\mathbf{f}$ and $\mathbf{c}$ are continuous functions, the existence of the solutions is guaranteed. Generally, uniqueness can be achieved by properly reducing the set $X$.

The assumption of well-posedness is particularly important for the coarse model and will be discussed later (Assumption 12).

\section{Manifold mapping}

\subsection{Original manifold mapping}

In [20] the manifold mapping $\mathbf{S}: \mathbf{c}(X) \rightarrow \mathbf{f}(X)$ is introduced with the aim of correcting the misalignment between the manifolds $\mathbf{f}(X)$ and $\mathbf{c}(X)$. By $\mathbf{S}$, the point $\mathbf{c}\left(\mathbf{x}_{\mathbf{f}}^{*}\right)$ is mapped to $\mathbf{f}\left(\mathbf{x}_{\mathbf{f}}^{*}\right)$ and the tangent plane for $\mathbf{c}(X)$ at $\mathbf{c}\left(\mathbf{x}_{\mathbf{f}}^{*}\right)$ to the tangent plane for $\mathbf{f}(X)$ at $\mathbf{f}\left(\mathbf{x}_{\mathbf{f}}^{*}\right)$ (see Fig. 1). Other approaches are possible but in this work we define $\mathbf{S}$ as the affine mapping

$\mathbf{S} \mathbf{c}(\mathbf{x})=\mathbf{f}\left(\mathbf{x}_{\mathbf{f}}^{*}\right)+\bar{S}\left(\mathbf{c}(\mathbf{x})-\mathbf{c}\left(\mathbf{x}_{\mathbf{f}}^{*}\right)\right)$,

where

$\bar{S}=J_{\mathbf{f}}\left(\mathbf{x}_{\mathbf{f}}^{*}\right) J_{\mathbf{c}}^{\dagger}\left(\mathbf{x}_{\mathbf{f}}^{*}\right)$.

Here the pseudo-inverse ${ }^{\dagger}$ is defined as $J_{\mathbf{c}}^{\dagger}\left(\mathbf{x}_{\mathbf{f}}^{*}\right)=V_{\mathbf{c}} \Sigma_{\mathbf{c}}^{\dagger} U_{\mathbf{c}}^{T}$, being $U_{\mathbf{c}}, \Sigma_{\mathbf{c}}$ and $V_{\mathbf{c}}$ the factors in the singular value decomposition of $J_{\mathbf{c}}\left(\mathbf{x}_{\mathbf{f}}^{*}\right)=U_{\mathbf{c}} \Sigma_{\mathbf{c}} V_{\mathbf{c}}^{T}$. The matrix $\Sigma_{\mathbf{c}}^{\dagger}$ is the result of inverting the nonzero entries in $\Sigma_{\mathfrak{c}}$, leaving the zeroes invariant. It should be noted that, because $J_{\mathbf{f}}\left(\mathbf{x}_{\mathbf{f}}^{*}\right)$ and $J_{\mathbf{c}}\left(\mathbf{x}_{\mathbf{f}}^{*}\right)$ are both full rank, also the $m \times m$ matrix $\bar{S}$ has rank $n$.

The manifold-mapping solution $\mathbf{x}_{\mathbf{m m}}^{*}$ is defined as

$\mathbf{x}_{\mathbf{m m}}^{*}=\underset{\mathbf{x} \in X}{\operatorname{argmin}}\|\mathbf{S c} \mathbf{c}(\mathbf{x})-\mathbf{y}\|$.

Now the combination $\mathbf{S} \circ \mathbf{c}$ acts as the surrogate model for $\mathbf{f}$ and under some assumptions about the similarity between the fine and the coarse model that usually hold in practice, it will be shown below that the manifold-mapping solution $\mathbf{x}_{\mathbf{m m}}^{*}$ is a local minimizer of the fine cost function. As one may expect, not every coarse model can be successfully used within the manifold-mapping framework. We partly formalize this by two assumptions:

Assumption 5 If $\|\mathbf{f}(\mathbf{x})-\mathbf{y}\|$ is locally convex at $\mathbf{x}_{\mathbf{f}}^{*}$ then $\|\mathbf{S ~ c}(\mathbf{x})-\mathbf{y}\|$ is also locally convex at $\mathbf{x}_{\mathbf{f}}^{*}$.

Assumption 6 If $\mathbf{x}_{\mathbf{f}}^{*}$ is a local minimizer of $\|\mathbf{S ~ c}(\mathbf{x})-\mathbf{y}\|$ then $\mathbf{x}_{\mathbf{f}}^{*}$ is the global optimum of $\|\mathbf{S ~ c}(\mathbf{x})-\mathbf{y}\|$.
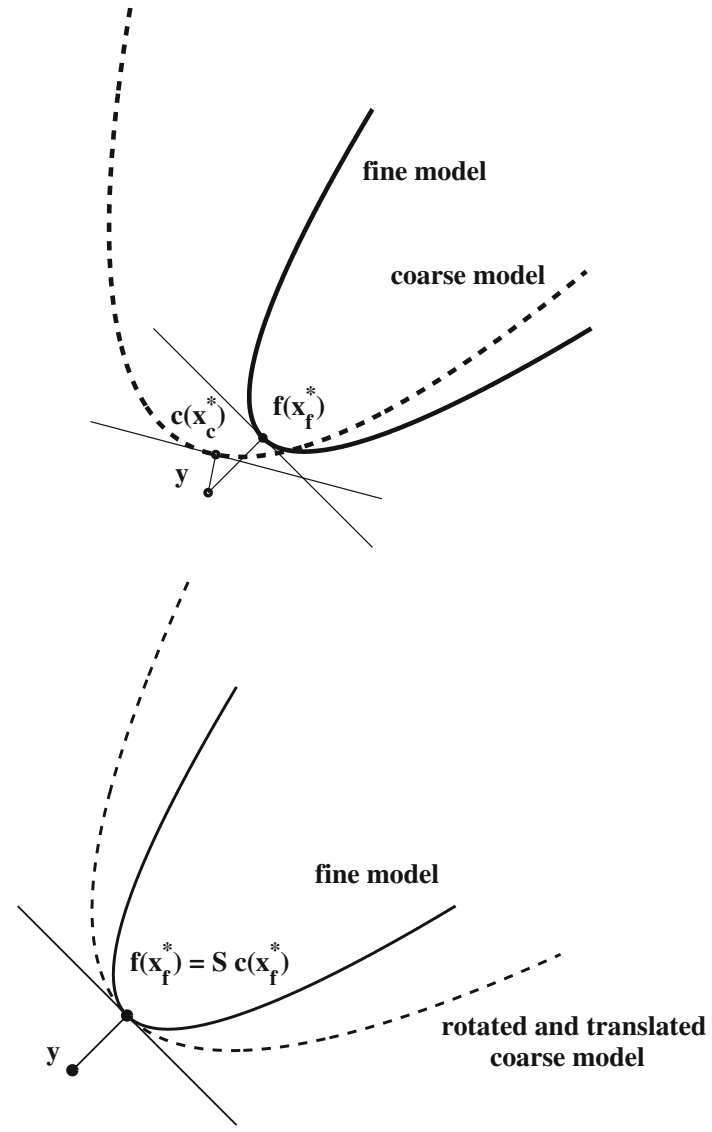

Fig. 1 Manifold-mapping model alignment

These are mild assumptions for the models used in practice. Assumption 5 specifies only a similar local behavior in the region of interest, i.e., in a neighborhood of the specifications $\mathbf{y}$. Assumption 6 means that the surrogate optimization does not introduce a spurious global optimum near the true minimizer $\mathbf{x}_{\mathbf{f}}^{*}$.

Lemma 1 Any (local) minimizer of the fine model cost function $\|\mathbf{f}(\mathbf{x})-\mathbf{y}\|$ is a (local) minimizer of $\|\mathbf{S} \mathbf{c}(\mathbf{x})-\mathbf{y}\|$.

Proof We denote a minimizer of the fine cost function by $\mathbf{x}_{\mathbf{f}}^{*}$. First we see that $\mathbf{x}_{\mathbf{f}}^{*}$ satisfies the KKT conditions associated with (16). From (14) and (15) we have $\mathbf{S ~ c}\left(\mathbf{x}_{\mathbf{f}}^{*}\right)=\mathbf{f}\left(\mathbf{x}_{\mathbf{f}}^{*}\right)$ and $J_{\mathbf{S} \mathbf{c}}\left(\mathbf{x}_{\mathbf{f}}^{*}\right)=\bar{S} J_{\mathbf{c}}\left(\mathbf{x}_{\mathbf{f}}^{*}\right)=J_{\mathbf{f}}\left(\mathbf{x}_{\mathbf{f}}^{*}\right)$. Thus, the first derivatives of $F(\mathbf{x})$ and of the surrogate cost function $\|\mathbf{S} \mathbf{c}(\mathbf{x})-\mathbf{y}\|$ coincide at $\mathbf{x}_{\mathbf{f}}^{*}$. Since the constraints are the same in both optimization problems and $\mathbf{x}_{\mathbf{f}}^{*}$ is a local minimizer of $F(\mathbf{x})$ (i.e., the fine KKT conditions hold), we conclude that $\mathbf{x}_{\mathbf{f}}^{*}$ satisfies the surrogate KKT conditions. The fine model cost function is locally convex at $\mathbf{x}_{\mathbf{f}}^{*}$. Because of Assumption 5, the fine model optimum is also a local minimizer of $\|\mathbf{S} \mathbf{c}(\mathbf{x})-\mathbf{y}\|$.

Lemma $2 \mathbf{x}_{\mathbf{m m}}^{*}$ is a local minimizer of $\|\mathbf{f}(\mathbf{x})-\mathbf{y}\|$.

Proof Use Lemma 1 and Assumption 6. 
Remark 2 We cannot directly conclude from Lemma 1 that $\mathbf{x}_{\mathbf{m m}}^{*}$ is a minimizer of the fine cost function because the point $\mathbf{x}_{\mathbf{f}}^{*}$ could be just a local minimizer of $\|\mathbf{S ~ c}(\mathbf{x})-\mathbf{y}\|$. This agrees with the way the two-level approach is taken into practice, as a local manifold correction.

Remark 3 Based on the proof for Lemma 1 we can think of a strategy for dealing with expensive constraints $\mathbf{k}_{\mathbf{f}}(\mathbf{x})$ and $\overline{\mathbf{k}}_{\mathbf{f}}(\mathbf{x})$, provided some fast-to-compute approximations $\mathbf{k}_{\mathbf{c}}(\mathbf{x})$ and $\overline{\mathbf{k}}_{\mathbf{c}}(\mathbf{x})$ are available. Analog manifold mappings for the constraints $\mathbf{S}_{\mathbf{k}}: \mathbf{k}_{\mathbf{c}}(X) \rightarrow \mathbf{k}_{\mathbf{f}}(X)$ and $\mathbf{S}_{\overline{\mathbf{k}}}: \overline{\mathbf{k}}_{\mathbf{c}}(X) \rightarrow \overline{\mathbf{k}}_{\mathbf{f}}(X)$ can be defined so that

$$
\begin{aligned}
& \mathbf{S}_{\mathbf{k}} \mathbf{k}_{\mathbf{c}}\left(\mathbf{x}_{\mathbf{f}}^{*}\right)=\mathbf{k}_{\mathbf{f}}\left(\mathbf{x}_{\mathbf{f}}^{*}\right), \\
& \mathbf{S}_{\overline{\mathbf{k}}} \overline{\mathbf{k}}_{\mathbf{c}}\left(\mathbf{x}_{\mathbf{f}}^{*}\right)=\overline{\mathbf{k}}_{\mathbf{f}}\left(\mathbf{x}_{\mathbf{f}}^{*}\right), \\
& J_{\mathbf{S}_{\mathbf{k}} \mathbf{k}_{\mathbf{c}}}\left(\mathbf{x}_{\mathbf{f}}^{*}\right)=J_{\mathbf{k}_{\mathbf{f}}}\left(\mathbf{x}_{\mathbf{f}}^{*}\right), \\
& J_{\mathbf{S}_{\overline{\mathbf{k}}} \overline{\mathbf{k}}_{\mathbf{c}}}\left(\mathbf{x}_{\mathbf{f}}^{*}\right)=J_{\overline{\mathbf{k}}_{\mathbf{f}}}\left(\mathbf{x}_{\mathbf{f}}^{*}\right),
\end{aligned}
$$

and thus again, the surrogate KKT conditions at $\mathbf{x}_{\mathbf{f}}^{*}$ reproduce the fine ones at the same point. This constraint manifold mapping is the approach taken in $[19,22]$.

The mapping $\mathbf{S}$ is not known a priori, because it depends on the solution of the optimization problem. We propose an algorithm (see Fig. 2) that, when it converges, yields-as we shall see- both the mapping $\mathbf{S}$ and the desired fine model optimum $\mathbf{x}_{\mathbf{f}}^{*}$. We will refer to this scheme as the original manifoldmapping (OMM) algorithm. The model alignment can be improved by an additional (right-preconditioning) mapping $\overline{\mathbf{p}}: X \rightarrow Z$. This mapping $\overline{\mathbf{p}}$ is optional in the present case where the coarse and fine control spaces $Z$ and $X$ coincide, but it is obligatory when those spaces differ. For simplicity, in the algorithm in Fig. 2 we take $\overline{\mathbf{p}}=I$, the identity. The optimization procedure needed to compute $\mathbf{x}_{k+1}$ is not essentially different from the one to obtain the coarse model optimum $\mathbf{x}_{\mathbf{c}}^{*}$. Therefore, we may expect the optimization problem to be well defined in each iteration step. However, formally this has to be introduced as an assumption.

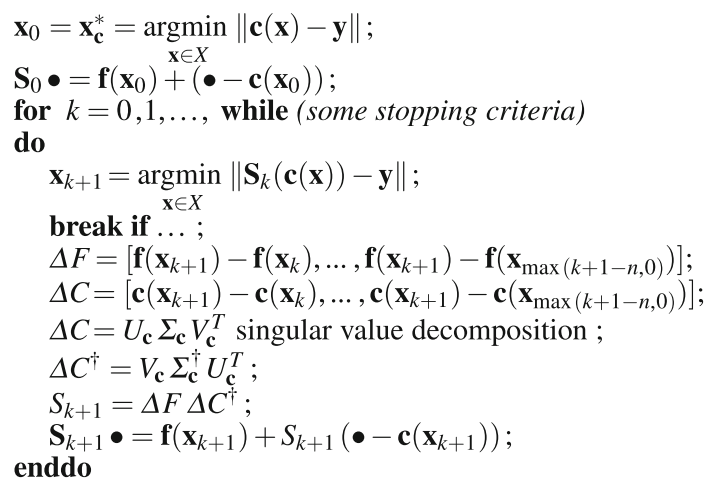

Fig. 2 The original manifold-mapping $(O M M)$ algorithm
Assumption 7 The minimization

$\mathbf{x}_{k+1}=\underset{\mathbf{x} \in X}{\operatorname{argmin}}\left\|\mathbf{S}_{k}(\mathbf{c}(\mathbf{x}))-\mathbf{y}\right\|$

is well defined for every $k$.

This assumption is the surrogate equivalent of Assumption 4.

For the proof that, if it converges, the OMM algorithm yields the fine model optimum, the following lemma will be very useful.

Lemma 3 Let $\widetilde{\mathbf{x}} \in X$ be the minimizer of a surrogate model problem

$\widetilde{\mathbf{x}}=\underset{\mathbf{x} \in X}{\operatorname{argmin}}\|\widetilde{\mathbf{S}} \mathbf{c}(\mathbf{x})-\mathbf{y}\|$,

with

$\widetilde{\mathbf{S}} \mathbf{c}(\mathbf{x})=\mathbf{f}(\widetilde{\mathbf{x}})+J_{\mathbf{f}}(\widetilde{\mathbf{x}}) J_{\mathbf{c}}^{\dagger}(\widetilde{\mathbf{x}})(\mathbf{c}(\mathbf{x})-\mathbf{c}(\widetilde{\mathbf{x}}))$,

where $\|\mathbf{f}(\mathbf{x})-\mathbf{y}\|$ is locally convex at $\widetilde{\mathbf{x}}$, then $\widetilde{\mathbf{x}}$ is a (local) minimizer of $\|\mathbf{f}(\mathbf{x})-\mathbf{y}\|$.

Proof Clearly, $\widetilde{\mathbf{x}}$ satisfies the KKT conditions associated with $\|\widetilde{\mathbf{S}} \mathbf{c}(\mathbf{x})-\mathbf{y}\|$, and because of (23) we have $\widetilde{\mathbf{S}} \mathbf{c}(\widetilde{\mathbf{x}})=\mathbf{f}(\widetilde{\mathbf{x}})$ and $J_{\widetilde{\mathbf{S}} \mathbf{c}}(\widetilde{\mathbf{x}})=J_{\mathbf{f}}(\widetilde{\mathbf{x}})$. Proceeding as in Lemma 1 we see that the point $\widetilde{\mathbf{x}}$ satisfies also the fine KKT conditions and, because $\|\mathbf{f}(\mathbf{x})-\mathbf{y}\|$ is locally convex at $\widetilde{\mathbf{x}}$, this point is a (local) minimizer of the fine cost function $F(\mathbf{x})$.

Remark 4 Note that from Lemma 3 it also follows that $\widetilde{\mathbf{S}}=\mathbf{S}$.

We can replace the requirement of $\|\mathbf{f}(\mathbf{x})-\mathbf{y}\|$ being locally convex at $\widetilde{\mathbf{x}}$ from Lemma 3 by an assumption, very similar in nature to Assumption 5, and also likely to hold in practice:

Assumption 8 If $\|\widetilde{\mathbf{S}} \mathbf{c}(\mathbf{x})-\mathbf{y}\|$ is locally convex at $\widetilde{\mathbf{x}}$, then $\|\mathbf{f}(\mathbf{x})-\mathbf{y}\|$ is locally convex at $\widetilde{\mathbf{x}}$.

Remark 5 The manifold-mapping theory is generally stated in terms of local alignment between the surrogate model and the fine model. As a consequence, we can only state results concerning local optima of the fine cost function.

Now we will show that, if the OMM algorithm in Fig. 2 converges to a fixed point $\overline{\mathbf{x}}$, this fixed point is a (local) minimizer of the fine cost function. The iterates of the OMM algorithm are denoted by $\mathbf{x}_{k}$. Because we are studying the fixed point situation, it may be assumed $k>n$.

Further, some additional mild assumptions are needed for proving that $\overline{\mathbf{x}}$ (locally) minimizes $\|\mathbf{f}(\mathbf{x})-\mathbf{y}\|$. Since the Jacobians $J_{\mathbf{f}}(\mathbf{x})$ and $J_{\mathbf{c}}(\mathbf{x})$ have both rank $n$, we expect that the matrices $\Delta F$ and $\Delta C$ in the OMM algorithm are also full-rank. In practice, this will generally be the case and for the exceptional situation where it is not, minor changes in the algorithm can be made with no real influence on the results. So, to prevent minor details in the discussion making the analysis much more complex, we introduce the following assumption. 
Assumption 9 For $k$ large enough, the $m \times n$ matrices $\Delta F$ and $\Delta C$ have rank $n$ and there are constants $K_{1}, K_{2}>0$ independent of $k$ such that

$$
\begin{aligned}
& \left(\max _{i=0, \ldots, n-1}\left\|\mathbf{x}_{k+1-i}-\overline{\mathbf{x}}\right\|^{2}\right)\left\|\Delta F^{\dagger}\right\|_{2}^{2} \leq K_{1}, \\
& \left(\max _{i=0, \ldots, n-1}\left\|\mathbf{x}_{k+1-i}-\overline{\mathbf{x}}\right\|^{2}\right)\left\|\Delta C^{\dagger}\right\|_{2}^{2} \leq K_{2} .
\end{aligned}
$$

We will see in Lemma 4 that Assumption 9, together with the next one below, guarantees that $\Delta F \Delta C^{\dagger}$ converges to $J_{\mathbf{f}}(\overline{\mathbf{x}}) J_{\mathbf{c}}^{\dagger}(\overline{\mathbf{x}})$ and thus that Lemma 3 can be applied.

Assumption 10 For $k$ large enough the matrix $\Delta X_{k+1}$ defined by

$\Delta X_{k+1}=\left[\mathbf{x}_{k+1}-\mathbf{x}_{k}, \mathbf{x}_{k+1}-\mathbf{x}_{k-1}, \ldots, \mathbf{x}_{k+1}-\mathbf{x}_{k-n+1}\right]$

is regular and there is a constant $K_{3}>0$ independent of $k$ such that

$$
\left(\max _{i=0, \ldots, n-1}\left\|\mathbf{x}_{k+1-i}-\overline{\mathbf{x}}\right\|^{2}\right)\left\|\Delta X_{k+1}^{-1}\right\|_{2}^{2} \leq K_{3} .
$$

Remark 6 Assumption 10 refers to the condition of the matrix $\Delta X_{k+1}$ and equivalently to the scaled step directions $\left(\mathbf{x}_{k+1-i}-\mathbf{x}_{k-i}\right.$, with $\left.i=0, \ldots, n-1\right)$. In the exceptional situations where the condition becomes too bad, the algorithm can be easily modified in order to alleviate that. Assumption 9 is related to Assumption 10 and to the wellposedness of the inverse model operators (see Assumption 12 concerning the coarse model). Because $\mathbf{c}(X)$ is a differentiable manifold we have $\Delta C \approx J_{\mathbf{c}}(\overline{\mathbf{x}}) \Delta X_{k+1}$ in a neighborhood of $\overline{\mathbf{x}}$, and thus, as it will become clear in the proof for Lemma 4 , that $\Delta C^{\dagger} \approx \Delta X_{k+1}^{-1} J_{\mathbf{c}}^{\dagger}(\overline{\mathbf{x}})$. Thus we can expect Assumption 9 to be satisfied if Assumption 10 holds and $\left\|J_{\mathbf{c}}^{\dagger}(\overline{\mathbf{x}})\right\|_{2}^{2}$ is bounded. This last fact can be expressed as the (general) inverse coarse model operator being Lipschitz in the region of interest. The inequality (24) is the analogous relation with respect to the fine model.

Lemma 4 Let the sequence of iterates $\mathbf{x}_{k}$ and operators $S_{k+1}$ be defined by the OMM algorithm. Then, under Assumptions 9 and 10 the operators $S_{k+1}$ converge to $J_{\mathbf{f}}(\overline{\mathbf{x}}) J_{\mathbf{c}}^{\dagger}(\overline{\mathbf{x}})$, where $\overline{\mathbf{x}}$ is the fixed point of the iteration.

Proof By Assumption 9 and because $\mathbf{f}$ and $\mathbf{c}$ are differentiable, we have

$$
\begin{aligned}
& \Delta F=J_{\mathbf{f}}(\overline{\mathbf{x}}) \Delta X_{k+1}+M_{\mathbf{f}} O\left(\max _{i=1, \ldots, n}\left\|\mathbf{x}_{k+1-i}-\overline{\mathbf{x}}\right\|^{2}\right), \\
& \Delta C=J_{\mathbf{c}}(\overline{\mathbf{x}}) \Delta X_{k+1}+M_{\mathbf{c}} O\left(\max _{i=1, \ldots, n}\left\|\mathbf{x}_{k+1-i}-\overline{\mathbf{x}}\right\|^{2}\right),
\end{aligned}
$$

where $M_{\mathbf{f}}$ and $M_{\mathbf{c}}$ are some $m \times n$ matrices that depend on the smoothness of the manifolds $\mathbf{f}(X)$ and $\mathbf{c}(X)$ but not on $k$. We can use a generalization of the Banach Lemma for the inverse of a perturbed matrix [24, Theorem 6.1-2] applied to $\Delta C$ and conclude that

$\left\|\Delta C^{\dagger}-\Delta X_{k+1}^{-1} J_{\mathbf{c}}^{\dagger}(\overline{\mathbf{x}})\right\|_{2} \leq 2\left\|M_{\mathbf{c}}\right\|_{2} \max \left\{\left\{K_{2}, K_{3}\left\|J_{\mathbf{c}}^{\dagger}(\overline{\mathbf{x}})\right\|_{2}^{2}\right\}\right.$.

Because of (28) and the fact that the norm of $\Delta C^{\dagger}-\Delta X_{k+1}^{-1} J_{\mathbf{c}}^{\dagger}$ $(\overline{\mathbf{x}})$ is bounded by a constant independent of $k$, we obtain that $S_{k+1}=\Delta F \Delta C^{\dagger}$ converges to $J_{\mathbf{f}}(\overline{\mathbf{x}}) J_{\mathbf{c}}^{\dagger}(\overline{\mathbf{x}})$.

Using this result, we can apply Lemma 3 and conclude that if the OMM algorithm converges, then the fixed point of the iteration is a local minimizer of the fine model cost function $\|\mathbf{f}(\mathbf{x})-\mathbf{y}\|$. This is summarized in the following theorem.

Theorem 1 Let $\overline{\mathbf{x}}$ be the fixed point of the OMM iteration (Fig. 2) and let the fine model cost function $F(\mathbf{x})=\|\mathbf{f}(\mathbf{x})-\mathbf{y}\|$ be locally convex at $\overline{\mathbf{x}}$, then under Assumptions 1, 2, 3, 4, 7, 9 and 10 the point $\overline{\mathbf{x}}$ is a local minimizer of $F(\mathbf{x})$.

Remark 7 The assumption of local convexity of $F(\mathbf{x})$ can be replaced by Assumption 8 (model similarity) with $\mathbf{S}$ as in (14), and (15).

Remark 8 The results thus far presented for the mapping proposed in the OMM algorithm can be extended, by the same arguments, to any such algorithm based on a matrix $\bar{S}$ satisfying $\bar{S} J_{\mathbf{c}}\left(\mathbf{x}_{\mathbf{f}}^{*}\right)=J_{\mathbf{f}}\left(\mathbf{x}_{\mathbf{f}}^{*}\right)$. In fact there is complete freedom how $\bar{S}$ handles components in the complement of the range of $U_{\mathbf{c}}$. The general case is $\bar{S}=J_{\mathbf{f}}\left(\mathbf{x}_{\mathbf{f}}^{*}\right) J_{\mathbf{c}}^{\dagger}\left(\mathbf{x}_{\mathbf{f}}^{*}\right)+A\left(I-U_{\mathbf{c}} U_{\mathbf{c}}^{T}\right)$ with $A$ any $m \times m$ matrix. This freedom can be used to stabilize the algorithm.

\subsection{Manifold mapping}

In the OMM algorithm, $\mathbf{S}_{k} \circ \mathbf{c}$ is used as the surrogate model, i.e., it is updated during iteration, and the aim $\mathbf{y}$ is kept constant. From an implementational point of view, it is interesting to proceed the other way round: the model is kept fixed and the aim is updated in each step. Then it is particularly attractive to take for this model the available coarse model, which is easily solved by assumption. This leads to a modification of the OMM algorithm. The procedure is shown in Fig. 3 and we denote it simply as the MM algorithm. As in the OMM algorithm we need an assumption to assure that $\mathbf{x}_{k+1}$ is well-defined:

Assumption 11 In the MM algorithm, the updated aims satisfy $\mathbf{y}_{k} \in Y \forall k$.

With this assumption replacing Assumption 7, we can prove for MM a theorem similar to Theorem 1 . 


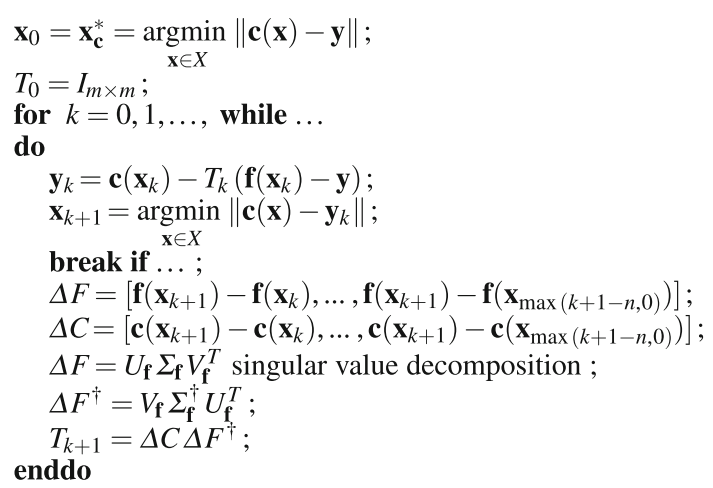

Fig. 3 The manifold-mapping $(M M)$ algorithm. Note that $T_{k}=S_{k}^{\dagger}$ for all $k>0$

Theorem 2 Let $\overline{\mathbf{x}}$ be the fixed point of the manifold-mapping iteration (MM, Fig. 3) and let the fine model cost function $F(\mathbf{x})=\|\mathbf{f}(\mathbf{x})-\mathbf{y}\|$ be locally convex at $\overline{\mathbf{x}}$, then under Assumptions 1, 2, 3, 4, 9, 10 and 11 the point $\overline{\mathbf{x}}$ is a local minimizer of $F(\mathbf{x})$.

Proof Proceeding as in Lemma 4 we can see that the sequence of operators $T_{k+1}=S_{k+1}^{\dagger}$ converges to $\left(J_{\mathbf{f}}(\overline{\mathbf{x}})\right.$ $\left.J_{\mathbf{c}}^{\dagger}(\overline{\mathbf{x}})\right)^{\dagger}=J_{\mathbf{c}}(\overline{\mathbf{x}}) J_{\mathbf{f}}^{\dagger}(\overline{\mathbf{x}})$. Also in the limit

$\overline{\mathbf{x}}=\underset{\mathbf{x} \in X}{\operatorname{argmin}}\left\|\mathbf{c}(\mathbf{x})-\mathbf{c}(\overline{\mathbf{x}})+\left(J_{\mathbf{f}}(\overline{\mathbf{x}}) J_{\mathbf{c}}^{\dagger}(\overline{\mathbf{x}})\right)^{\dagger}(\mathbf{f}(\overline{\mathbf{x}})-\mathbf{y})\right\|$.

Since $\mathbf{c}(X)$ is a manifold of class $C^{2},(31)$ is equivalent to

$\overline{\mathbf{x}}=\underset{\mathbf{x} \in X}{\operatorname{argmin}}\left\|J_{\mathbf{c}}(\overline{\mathbf{x}})(\mathbf{x}-\overline{\mathbf{x}})+\left(J_{\mathbf{f}}(\overline{\mathbf{x}}) J_{\mathbf{c}}^{\dagger}(\overline{\mathbf{x}})\right)^{\dagger}(\mathbf{f}(\overline{\mathbf{x}})-\mathbf{y})\right\|$.

Because $J_{\mathbf{c}}(\overline{\mathbf{x}})$ and $J_{\mathbf{f}}(\overline{\mathbf{x}})$ are full rank, we can write the former equality as

$\overline{\mathbf{x}}=\underset{\mathbf{x} \in X}{\operatorname{argmin}}\left\|J_{\mathbf{f}}(\overline{\mathbf{x}}) J_{\mathbf{c}}^{\dagger}(\overline{\mathbf{x}}) J_{\mathbf{c}}(\overline{\mathbf{x}})(\mathbf{x}-\overline{\mathbf{x}})+\mathbf{f}(\overline{\mathbf{x}})-\mathbf{y}\right\|$.

And with the same reasoning as in (31)-(32), equation (33) is equivalent to

$\overline{\mathbf{x}}=\underset{\mathbf{x} \in X}{\operatorname{argmin}}\left\|J_{\mathbf{f}}(\overline{\mathbf{x}}) J_{\mathbf{c}}^{\dagger}(\overline{\mathbf{x}})(\mathbf{c}(\mathbf{x})-\mathbf{c}(\overline{\mathbf{x}}))+\mathbf{f}(\overline{\mathbf{x}})-\mathbf{y}\right\|$.

Then Lemma 3 is applied in order to show that $\overline{\mathbf{x}}$ is a local minimizer of $\|\mathbf{f}(\mathbf{x})-\mathbf{y}\|$.

As a consequence of this theorem and by just rewriting (31) with $\overline{\mathbf{x}}$ a local minimizer of the fine cost function $F(\mathbf{x})$, the following interesting property for the fine model optimum can be formulated.

\section{Corollary 1 The fixed point of the MM iteration $\mathbf{x}_{\mathbf{f}}^{*}$ satisfies}

$\mathbf{x}_{\mathbf{f}}^{*}=\underset{\mathbf{x} \in X}{\operatorname{argmin}}\left\|\mathbf{c}(\mathbf{x})-\mathbf{c}\left(\mathbf{x}_{\mathbf{f}}^{*}\right)+\bar{S}^{\dagger}\left(\mathbf{f}\left(\mathbf{x}_{\mathbf{f}}^{*}\right)-\mathbf{y}\right)\right\|$.
In Sect. 4 we show that both schemes, OMM and MM, behave asymptotically the same and that the convergence study for these two algorithms coincides.

Remark 9 By a reasoning similar to that in Remark 8 it can be shown that the manifold-mapping algorithm can be based on any matrix $\bar{S}$ satisfying $J_{\mathbf{c}}^{\dagger}\left(\mathbf{x}_{\mathbf{f}}^{*}\right) \bar{S}^{\dagger}=J_{\mathbf{f}}^{\dagger}\left(\mathbf{x}_{\mathbf{f}}^{*}\right)$. In fact there is complete freedom with respect to how $\bar{S}$ handles components in the complement of the range of $U_{\mathbf{c}}$. The general case is $\bar{S}^{\dagger}=J_{\mathbf{c}}\left(\mathbf{x}_{\mathbf{f}}^{*}\right) J_{\mathbf{f}}^{\dagger}\left(\mathbf{x}_{\mathbf{f}}^{*}\right)+\left(I-U_{\mathbf{c}} U_{\mathbf{c}}^{T}\right) A$ with $A$ any $m \times m$ matrix. In [20], the particular cases $A=\left(I-U_{\mathbf{f}} U_{\mathbf{f}}^{T}\right)$ and $A=I$ are considered.

\subsection{Generalized manifold mapping}

The two algorithms, OMM and MM, can be generalized by selecting any $S_{k}$ for which Lemma 4 holds (i.e., $S_{k}$ converges to $\left.J_{\mathbf{f}}(\overline{\mathbf{x}}) J_{\mathbf{c}}^{\dagger}(\overline{\mathbf{x}})\right)$. We call this more general scheme generalized manifold mapping (GMM). A natural choice for $S_{k}$ would be $J_{\mathbf{f}}\left(\mathbf{x}_{k}\right) J_{\mathbf{c}}^{\dagger}\left(\mathbf{x}_{k}\right)$. This makes sense in particular in case the Jacobian of the fine model is available, e.g., via automatic differentiation [25] or by some adjoint-based technique [32]. But in most cases that information cannot be obtained efficiently and, therefore, we rather write $S_{k}=\widetilde{J}_{\mathbf{f}}\left(\mathbf{x}_{k}\right) J_{\mathbf{c}}^{\dagger}\left(\mathbf{x}_{k}\right)$, with $\widetilde{J}_{\mathbf{f}}\left(\mathbf{x}_{k}\right)$ an approximation of $J_{\mathbf{f}}\left(\mathbf{x}_{k}\right)$, e.g., computed by means of Broyden's method [14]. In Fig. 4 the GMM scheme corresponding to the $\mathrm{MM}$ algorithm is shown. If an approximation for $\widetilde{J}_{\mathbf{f}}\left(\mathbf{x}_{0}\right)$ is available, $T_{0}=J_{\mathbf{c}}\left(\mathbf{x}_{0}\right) \widetilde{J}_{\mathbf{f}}\left(\mathbf{x}_{0}\right)$ could be used as initial guess instead of the identity matrix. We consider Assumption 11 to be also applicable to GMM.

Under convergence of the GMM scheme, we can also see that again the fixed point for the iteration is the solution of the optimization problem, $\mathbf{x}_{\mathbf{f}}^{*}$. The proof for the following theorem is completely analogous to those given for Theorems 1 and 2 and is, therefore, omitted.

Theorem 3 Let $\overline{\mathbf{x}}$ be the fixed point of the GMM algorithms (Fig. 4) and let the fine model cost function $F(\mathbf{x})=\|\mathbf{f}(\mathbf{x})-\mathbf{y}\|$ be locally convex at $\overline{\mathbf{x}}$, then under Assumptions 1, 2, 3, 4, 9, 10 and 11 the point $\overline{\mathbf{x}}$ is a local minimizer of $F(\mathbf{x})$.

$$
\begin{aligned}
& \quad \mathbf{x}_{0}=\mathbf{x}_{\mathbf{c}}^{*}=\underset{\mathbf{x} \in X}{\operatorname{argmin}}\|\mathbf{c}(\mathbf{x})-\mathbf{y}\| ; \\
& T_{0}=I_{m \times m} ; \ldots, \text { while } \ldots \\
& \text { for } k=0,1, \ldots, \\
& \text { do } \quad \mathbf{y}_{k}=\mathbf{c}\left(\mathbf{x}_{k}\right)-T_{k}\left(\mathbf{f}\left(\mathbf{x}_{k}\right)-\mathbf{y}\right) ; \\
& \quad \mathbf{x}_{k+1}=\underset{\mathbf{x} \in X}{\operatorname{argmin}}\left\|\mathbf{c}(\mathbf{x})-\mathbf{y}_{k}\right\| ; \\
& \quad \text { break if } \ldots ; \\
& \quad T_{k+1}=J_{\mathbf{c}}\left(\mathbf{x}_{k+1}\right) \widetilde{J}_{\mathbf{f}}^{\dagger}\left(\mathbf{x}_{k+1}\right) ; \\
& \text { enddo }
\end{aligned}
$$

Fig. 4 The generalized manifold-mapping $(G M M)$ algorithm. Note that $T_{k}=S_{k}^{\dagger}$ for all $k>0$. $\widetilde{J}_{\mathbf{f}}\left(\mathbf{x}_{k+1}\right)$ is either $J_{\mathbf{f}}\left(\mathbf{x}_{k+1}\right)$ in case it is available or, in other cases, it is an estimate of it 


\section{Manifold-mapping convergence proof}

Here we first present conditions for convergence of the MM algorithm and then we show that the OMM iteration can be written in such a way that an equivalent convergence theorem can be stated for it, with a proof analogous to that for MM.

We define the general inverse coarse operator $\mathbf{c}^{\dagger}: Y \subset$ $\mathbb{R}^{m} \rightarrow X \subset \mathbb{R}^{n}$ by

$\mathbf{c}^{\dagger} \overline{\mathbf{y}}=\underset{\mathbf{x} \in X}{\operatorname{argmin}}\|\mathbf{c}(\mathbf{x})-\overline{\mathbf{y}}\|$.

We notice that this operator is well-defined because of Assumption 4. The coarse model is an essential component of any manifold-mapping technique. We are interested in coarse models whose associated inverse operators are well-posed. This is formalized in the next condition that should be satisfied by any coarse model used in practice.

Assumption 12 The general inverse coarse model operator, $\mathbf{c}^{\dagger}$, is Lipschitz with a Lipschitz constant bounded by $L_{\mathbf{c}^{\dagger}}$.

We can write (35) and the expression for iterate $\mathbf{x}_{k+1}$ in the MM algorithm as

$\mathbf{x}_{\mathbf{f}}^{*}=\mathbf{c}^{\dagger}\left(\mathbf{c}\left(\mathbf{x}_{\mathbf{f}}^{*}\right)-\bar{S}^{\dagger}\left(\mathbf{f}\left(\mathbf{x}_{\mathbf{f}}^{*}\right)-\mathbf{y}\right)\right)$,

$\mathbf{x}_{k+1}=\mathbf{c}^{\dagger}\left(\mathbf{c}\left(\mathbf{x}_{k}\right)-S_{k}^{\dagger}\left(\mathbf{f}\left(\mathbf{x}_{k}\right)-\mathbf{y}\right)\right)$.

Subtracting (37) from (38) and using Assumption 12, we get

$$
\begin{aligned}
& \left\|\mathbf{x}_{k+1}-\mathbf{x}_{\mathbf{f}}^{*}\right\| \\
& \quad \leq L_{\mathbf{c}^{\dagger}}\left\|\mathbf{c}\left(\mathbf{x}_{k}\right)-S_{k}^{\dagger}\left(\mathbf{f}\left(\mathbf{x}_{k}\right)-\mathbf{y}\right)-\mathbf{c}\left(\mathbf{x}_{\mathbf{f}}^{*}\right)+\bar{S}^{\dagger}\left(\mathbf{f}\left(\mathbf{x}_{\mathbf{f}}^{*}\right)-\mathbf{y}\right)\right\| .
\end{aligned}
$$

We can write the expression in the norm at the right-hand side as

$$
\begin{aligned}
\mathbf{c}\left(\mathbf{x}_{k}\right)-S_{k}^{\dagger}\left(\mathbf{f}\left(\mathbf{x}_{k}\right)-\mathbf{y}\right)-\mathbf{c}\left(\mathbf{x}_{\mathbf{f}}^{*}\right)+\bar{S}^{\dagger}\left(\mathbf{f}\left(\mathbf{x}_{\mathbf{f}}^{*}\right)-\mathbf{y}\right) \\
=\mathbf{c}\left(\mathbf{x}_{k}\right)-\mathbf{c}\left(\mathbf{x}_{\mathbf{f}}^{*}\right)-S_{k}^{\dagger}\left(\mathbf{f}\left(\mathbf{x}_{k}\right)-\mathbf{f}\left(\mathbf{x}_{\mathbf{f}}^{*}\right)\right)+\left(\bar{S}^{\dagger}-S_{k}^{\dagger}\right)\left(\mathbf{f}\left(\mathbf{x}_{\mathbf{f}}^{*}\right)-\mathbf{y}\right) \\
=\left(J_{\mathbf{c}}\left(\mathbf{x}_{\mathbf{f}}^{*}\right)-S_{k}^{\dagger} J_{\mathbf{f}}\left(\mathbf{x}_{\mathbf{f}}^{*}\right)\right)\left(\mathbf{x}_{k}-\mathbf{x}_{\mathbf{f}}^{*}\right)+\left(\bar{S}^{\dagger}-S_{k}^{\dagger}\right)\left(\mathbf{f}\left(\mathbf{x}_{\mathbf{f}}^{*}\right)-\mathbf{y}\right) \\
\quad+O\left(\left\|\mathbf{x}_{k}-\mathbf{x}_{\mathbf{f}}^{*}\right\|^{2}\right) .
\end{aligned}
$$

We now analyze the term $\left(\bar{S}^{\dagger}-S_{k}^{\dagger}\right)$ in (40). We proceed as in the proof of Lemma 4 , with the difference that we cannot assume convergence of the algorithm. Since we know the possible fixed point of the iteration, with assumptions analogous to those for Lemma 4 (Assumptions 9 and 10) we will be able to establish a relation between $\left\|\bar{S}^{\dagger}-S_{k}^{\dagger}\right\|$ and $\left\|\mathbf{x}_{\mathbf{f}}^{*}-\mathbf{x}_{k}\right\|$.

Assumption 13 For $k$ large enough, there are constants $K_{4}$, $K_{5}>0$ independent of $k$ such that

$$
\begin{aligned}
& \left(\max _{i=1, \ldots, n}\left\|\mathbf{x}_{k+1-i}-\mathbf{x}_{k+1}\right\|^{2}\right)\left\|\Delta F^{\dagger}\right\|_{2}^{2} \leq K_{4}, \\
& \left(\max _{i=1, \ldots, n}\left\|\mathbf{x}_{k+1-i}-\mathbf{x}_{k+1}\right\|^{2}\right)\left\|\Delta C^{\dagger}\right\|_{2}^{2} \leq K_{5} .
\end{aligned}
$$

Assumption 14 For $k$ large enough, there is a constant $K_{6}>$ 0 independent of $k$ such that

$$
\left(\max _{i=1, \ldots, n}\left\|\mathbf{x}_{k+1-i}-\mathbf{x}_{k+1}\right\|^{2}\right)\left\|\Delta X_{k+1}^{-1}\right\|_{2}^{2} \leq K_{6},
$$

where $\Delta X_{k+1}$ is the square matrix defined in Assumption 10 .

Remark 10 We recognize in

$\left(\max _{i=1, \ldots, n}\left\|\mathbf{x}_{k+1-i}-\mathbf{x}_{k+1}\right\|^{2}\right)^{1 / 2}$

a matrix-norm for $\Delta X_{k+1}$. Thus, Assumption 14 can be stated in terms of $\kappa\left(\Delta X_{k+1}\right)$ the condition number of $\Delta X_{k+1}$, i.e., $\kappa\left(\Delta X_{k+1}\right) \leq K_{6}$, with $K_{6}>0$ a constant independent of $k$. In the rare situations where linear dependence in the step directions is obtained, the algorithm can be slightly modified, with no significant influence in the final result, to cope with that issue [27]. Assumption 13 can be related to the condition number of $\Delta X_{k+1}$ and the well-posedness of the inverse model operators in the region of interest.

Lemma 5 Under Assumptions 1, 2, 3, 4, 8, 9, 10, 11, 13 and 14, we find

$$
\left\|S_{k}^{\dagger}-J_{\mathbf{c}}\left(\mathbf{x}_{k}\right) J_{\mathbf{f}}^{\dagger}\left(\mathbf{x}_{k}\right)\right\| \leq M_{1} \max _{i=0, \ldots, n-1}\left\|\mathbf{x}_{k-i}-\mathbf{x}_{\mathbf{f}}^{*}\right\|,
$$

where $M_{1}>0$ is a constant that depends on the smoothness of the manifolds $\mathbf{f}(X)$ and $\mathbf{c}(X)$ but not on $k$.

Proof As in Lemma 4 we can write

$$
\begin{aligned}
\Delta F & =J_{\mathbf{f}}\left(\mathbf{x}_{k+1}\right) \Delta X_{k+1}+M_{\mathbf{f}} O\left(\max _{i=1, \ldots, n}\left\|\mathbf{x}_{k+1-i}-\mathbf{x}_{k+1}\right\|^{2}\right), \\
\Delta C & =J_{\mathbf{c}}\left(\mathbf{x}_{k+1}\right) \Delta X_{k+1}+M_{\mathbf{c}} O\left(\max _{i=1, \ldots, n}\left\|\mathbf{x}_{k+1-i}-\mathbf{x}_{k+1}\right\|^{2}\right),
\end{aligned}
$$

where $M_{\mathbf{f}}$ and $M_{\mathbf{c}}$ are some $m \times n$ matrices that depend on the smoothness of the manifolds $\mathbf{f}(X)$ and $\mathbf{c}(X)$ but not on $k$. Again with [24, Theorem 6.1-2] and by Assumptions 13 and 14 we can conclude that $S_{k}^{\dagger}-J_{\mathbf{c}}\left(\mathbf{x}_{k}\right) J_{\mathbf{f}}^{\dagger}\left(\mathbf{x}_{k}\right)$ is bounded by a constant multiplied by $\max _{i=1, \ldots, n}\left\|\mathbf{x}_{k-i}-\mathbf{x}_{k}\right\|$. Clearly, this is equivalent to $S_{k}^{\dagger}-J_{\mathbf{c}}\left(\mathbf{x}_{k}\right) J_{\mathbf{f}}^{\dagger}\left(\mathbf{x}_{k}\right)$ being bounded by $M_{1} \max _{i=0, \ldots, n-1}\left\|\mathbf{x}_{k-i}-\mathbf{x}_{\mathbf{f}}^{*}\right\|$ where $M_{1}>0$ is a constant that depends on the smoothness of $\mathbf{f}(X)$ and $\mathbf{c}(X)$ but not on $k$.

Remark 11 The constant $M_{1}$ depends on the smoothness of $\mathbf{f}(X)$ and $\mathbf{c}(X)\left(M_{1}=0\right.$ if both manifolds are linear in the neighborhood of the solution). 
Lemma 6 Under Assumptions 1, 2, 3, 4, 8, 9, 10, 11, 13 and 14, we find

$\left\|\bar{S}^{\dagger}-J_{\mathbf{c}}\left(\mathbf{x}_{k}\right) J_{\mathbf{f}}^{\dagger}\left(\mathbf{x}_{k}\right)\right\| \leq M_{2}\left\|\mathbf{x}_{k}-\mathbf{x}_{\mathbf{f}}^{*}\right\|$,

where $M_{2}>0$ is a constant that depends on the smoothness of the manifolds $\mathbf{f}(X)$ and $\mathbf{c}(X)$ but not on $k$.

Proof We can write

$$
\begin{aligned}
\bar{S}^{\dagger}-J_{\mathbf{c}}\left(\mathbf{x}_{k}\right) J_{\mathbf{f}}^{\dagger}\left(\mathbf{x}_{k}\right)= & J_{\mathbf{c}}\left(\mathbf{x}_{\mathbf{f}}^{*}\right) J_{\mathbf{f}}^{\dagger}\left(\mathbf{x}_{\mathbf{f}}^{*}\right)-J_{\mathbf{c}}\left(\mathbf{x}_{k}\right) J_{\mathbf{f}}^{\dagger}\left(\mathbf{x}_{k}\right) \\
= & J_{\mathbf{c}}\left(\mathbf{x}_{\mathbf{f}}^{*}\right) J_{\mathbf{f}}^{\dagger}\left(\mathbf{x}_{\mathbf{f}}^{*}\right)-J_{\mathbf{c}}\left(\mathbf{x}_{k}\right) J_{\mathbf{f}}^{\dagger}\left(\mathbf{x}_{\mathbf{f}}^{*}\right) \\
& +J_{\mathbf{c}}\left(\mathbf{x}_{k}\right) J_{\mathbf{f}}^{\dagger}\left(\mathbf{x}_{\mathbf{f}}^{*}\right)-J_{\mathbf{c}}\left(\mathbf{x}_{k}\right) J_{\mathbf{f}}^{\dagger}\left(\mathbf{x}_{k}\right) \\
= & \left(J_{\mathbf{c}}\left(\mathbf{x}_{\mathbf{f}}^{*}\right)-J_{\mathbf{c}}\left(\mathbf{x}_{k}\right)\right) J_{\mathbf{f}}^{\dagger}\left(\mathbf{x}_{\mathbf{f}}^{*}\right) \\
& +J_{\mathbf{c}}\left(\mathbf{x}_{k}\right)\left(J_{\mathbf{f}}^{\dagger}\left(\mathbf{x}_{\mathbf{f}}^{*}\right)-J_{\mathbf{f}}^{\dagger}\left(\mathbf{x}_{k}\right)\right) .
\end{aligned}
$$

Since $\mathbf{f}(X)$ and $\mathbf{c}(X)$ are manifolds of class $C^{2}$ and the (general) inverse operators are Lipschitz, we can bound $\| \bar{S}^{\dagger}$ $J_{\mathbf{c}}\left(\mathbf{x}_{k}\right) J_{\mathbf{f}}^{\dagger}\left(\mathbf{x}_{k}\right) \|$ by $M_{2}\left\|\mathbf{x}_{k}-\mathbf{x}_{\mathbf{f}}^{*}\right\|$, where $M_{2}>0$ is a constant that depends on the smoothness of the two manifolds but not on $k$.

Corollary 2 Under Assumptions 1, 2, 3, 4, 8, 9, 10, 11, 13 and 14 , we have

$\left\|\bar{S}^{\dagger}-S_{k}^{\dagger}\right\| \leq M \max _{i=0, \ldots, n-1}\left\|\mathbf{x}_{k-i}-\mathbf{x}_{\mathbf{f}}^{*}\right\|$,

where $M>0$ is a constant that depends on the smoothness of the manifolds $\mathbf{f}(X)$ and $\mathbf{c}(X)$ but not on $k$.

Proof We apply Lemmas 5 and 6 and set $M=\max \left(M_{1}, M_{2}\right)$.

Now, combining (39) and (40) we get

$$
\begin{aligned}
\left\|\mathbf{x}_{k+1}-\mathbf{x}_{\mathbf{f}}^{*}\right\| \leq & L_{\mathbf{c}^{\dagger}}\left\|J_{\mathbf{c}}\left(\mathbf{x}_{\mathbf{f}}^{*}\right)-S_{k}^{\dagger} J_{\mathbf{f}}\left(\mathbf{x}_{\mathbf{f}}^{*}\right)\right\|\left\|\mathbf{x}_{k}-\mathbf{x}_{\mathbf{f}}^{*}\right\| \\
& +\left\|\bar{S}^{\dagger}-S_{k}^{\dagger}\right\|\left\|\mathbf{f}\left(\mathbf{x}_{\mathbf{f}}^{*}\right)-\mathbf{y}\right\|+O\left(\left\|\mathbf{x}_{k}-\mathbf{x}_{\mathbf{f}}^{*}\right\|^{2}\right) .
\end{aligned}
$$

Due to (47) we can finally write

$$
\begin{aligned}
\left\|\mathbf{x}_{k+1}-\mathbf{x}_{\mathbf{f}}^{*}\right\| \leq & L_{\mathbf{c}^{\dagger}}\left(\left\|J_{\mathbf{c}}\left(\mathbf{x}_{\mathbf{f}}^{*}\right)-S_{k}^{\dagger} J_{\mathbf{f}}\left(\mathbf{x}_{\mathbf{f}}^{*}\right)\right\|\right. \\
& \left.+M\left\|\mathbf{f}\left(\mathbf{x}_{\mathbf{f}}^{*}\right)-\mathbf{y}\right\|\right) \max _{i=0, \ldots, n-1}\left\|\mathbf{x}_{k-i}-\mathbf{x}_{\mathbf{f}}^{*}\right\| \\
& +O\left(\left\|\mathbf{x}_{k}-\mathbf{x}_{\mathbf{f}}^{*}\right\|^{2}\right) .
\end{aligned}
$$

We formulate this result in the following theorem.

Theorem 4 Under Assumptions 1, 2, 3, 4, 8, 9, 10, 11, 12, 13 and 14 , and the condition

$L_{\mathbf{c}^{\dagger}}\left(\left\|J_{\mathbf{c}}\left(\mathbf{x}_{\mathbf{f}}^{*}\right)-S_{k}^{\dagger} J_{\mathbf{f}}\left(\mathbf{x}_{\mathbf{f}}^{*}\right)\right\|+M\left\|\mathbf{f}\left(\mathbf{x}_{\mathbf{f}}^{*}\right)-\mathbf{y}\right\|\right)<1$ for $k \geq k_{0}$,

where $M>0$ is a constant that depends on the smoothness of the manifolds $\mathbf{f}(X)$ and $\mathbf{c}(X)$ but not on $k$, the manifoldmapping algorithm (MM, Fig. 3) yields (linear) convergence to $\mathbf{x}_{\mathbf{f}}^{*}$.
Corollary 3 If in addition to the assumptions for Theorem 4 we have $\mathbf{f}\left(\mathbf{x}_{\mathbf{f}}^{*}\right)=\mathbf{y}$ (i.e., a reachable design), then the convergence of the MM algorithm is superlinear.

Corollary 4 If in addition to the assumptions for Theorem 4 we have $S_{k}=\bar{S}=J_{\mathbf{f}}\left(\mathbf{x}_{\mathbf{f}}^{*}\right) J_{\mathbf{c}}^{\dagger}\left(\mathbf{x}_{\mathbf{f}}^{*}\right)$ for every $k \geq k_{0}$, then the convergence of the MM algorithm is quadratic.

As a corollary of the following two lemmas, we will see that - under convergence - the original (OMM) and the MM iterations are asymptotically equivalent.

Lemma 7 If the iteration in the OMM algorithm converges, for a large enough $k$ we find for its iterate $\mathbf{x}_{k+1}$

$$
\begin{aligned}
& \mathbf{x}_{k+1} \\
& \quad=\underset{\mathbf{x} \in X}{\operatorname{argmin}}\left\|\mathbf{c}(\mathbf{x})-\mathbf{c}\left(\mathbf{x}_{k}\right)+S_{k}^{\dagger}\left(\mathbf{f}\left(\mathbf{x}_{k}\right)-\mathbf{y}\right)+O\left(\left\|\mathbf{x}-\mathbf{x}_{k}\right\|^{2}\right)\right\| .
\end{aligned}
$$

Proof Because $\mathbf{c}(X)$ is differentiable in a neighborhood of the fixed point, for a large enough $k$ we can write

$$
\begin{aligned}
& \mathbf{x}_{k+1}=\underset{\mathbf{x} \in X}{\operatorname{argmin}}\left\|S_{k} \mathbf{c}(\mathbf{x})-S_{k} \mathbf{c}\left(\mathbf{x}_{k}\right)+\mathbf{f}\left(\mathbf{x}_{k}\right)-\mathbf{y}\right\| \\
&=\underset{\mathbf{x} \in X}{\operatorname{argmin}} \| S_{k} J_{\mathbf{c}}\left(\mathbf{x}_{k}\right)\left(\mathbf{x}-\mathbf{x}_{k}\right) \\
&+\mathbf{f}\left(\mathbf{x}_{k}\right)-\mathbf{y}+S_{k} O\left(\left\|\mathbf{x}-\mathbf{x}_{k}\right\|^{2}\right) \| \\
&= \underset{\mathbf{x} \in X}{\operatorname{argmin}} \| S_{k} J_{\mathbf{c}}\left(\mathbf{x}_{k}\right)\left(\mathbf{x}-\mathbf{x}_{k}\right) \\
&+\mathbf{f}\left(\mathbf{x}_{k}\right)-\mathbf{y}+O\left(\left\|\mathbf{x}-\mathbf{x}_{k}\right\|^{2}\right) \|,
\end{aligned}
$$

and for the last equality we remember that $S_{k}$ converges to $\bar{S}$ (Lemma 4). The iterate $\mathbf{x}_{k+1}$ can be expressed as

$$
\begin{array}{r}
\mathbf{x}_{k+1}=\underset{\mathbf{x} \in X}{\operatorname{argmin}} \| S_{k} \Delta C \Delta X_{k}^{-1}\left(\mathbf{x}-\mathbf{x}_{k}\right) \\
+\mathbf{f}\left(\mathbf{x}_{k}\right)-\mathbf{y}+O\left(\left\|\mathbf{x}-\mathbf{x}_{k}\right\|^{2}\right) \|,
\end{array}
$$

where, for a large enough $k$ we have $O\left(\left\|\mathbf{x}-\mathbf{x}_{k}\right\|\right)=$ $O\left(\max _{i=1, \ldots, n}\left\|\mathbf{x}_{k-i}-\mathbf{x}_{k}\right\|\right)$ in the Taylor expansions, since there is convergence. Because $S_{k}=\Delta F \Delta C^{\dagger}$ and $\Delta C^{\dagger} \Delta C=I$, we have

$$
\begin{aligned}
\mathbf{x}_{k+1}= & \underset{\mathbf{x} \in X}{\operatorname{argmin}} \| \Delta F \Delta X_{k}^{-1}\left(\mathbf{x}-\mathbf{x}_{k}\right) \\
& +\mathbf{f}\left(\mathbf{x}_{k}\right)-\mathbf{y}+O\left(\left\|\mathbf{x}-\mathbf{x}_{k}\right\|^{2}\right) \| .
\end{aligned}
$$

Further, since $\Delta F$ and $\Delta C$ are full-rank and $\Delta X_{k}$ is regular

$$
\begin{aligned}
\mathbf{x}_{k+1}= & \underset{\mathbf{x} \in X}{\operatorname{argmin}} \| \Delta C \Delta X_{k}^{-1}\left(\mathbf{x}-\mathbf{x}_{k}\right) \\
& +S_{k}^{\dagger}\left(\mathbf{f}\left(\mathbf{x}_{k}\right)-\mathbf{y}\right)+O\left(\left\|\mathbf{x}-\mathbf{x}_{k}\right\|^{2}\right) \| .
\end{aligned}
$$

The lemma follows immediately from this last equation.

Lemma 8 If the iteration in the MM algorithm converges, for a large enough $k$ we find for its iterate $\mathbf{x}_{k+1}$

$$
\begin{aligned}
\mathbf{x}_{k+1}= & \underset{\mathbf{x} \in X}{\operatorname{argmin}} \| S_{k}\left(\mathbf{c}(\mathbf{x})-\mathbf{c}\left(\mathbf{x}_{k}\right)\right)+\mathbf{f}\left(\mathbf{x}_{k}\right)-\mathbf{y} \\
& +O\left(\left\|\mathbf{x}-\mathbf{x}_{k}\right\|^{2}\right) \| .
\end{aligned}
$$

Proof Analogous to that for Lemma 7. 
Corollary 5 Under convergence, the OMM algorithm and the MM algorithm are asymptotically equivalent.

Remark 12 Due to Corollary 5, Theorem 4 and Corollaries 3 and 4 are also valid for the OMM (Fig. 2) if Assumption 11 is replaced by Assumption 7 .

Remark 13 By similar arguments as given for mappings based on $\bar{S}=J_{\mathbf{f}}\left(\mathbf{x}_{\mathbf{f}}^{*}\right) J_{\mathbf{c}}^{\dagger}\left(\mathbf{x}_{\mathbf{f}}^{*}\right)$, analogous convergence results can be obtained for the other versions of the algorithms as introduced in Remarks 8 and 9.

\subsection{GMM convergence proof}

The proof for GMM is analogous to that for MM (by construction of $S_{k+1}$ we fulfill the conditions for Lemma 5). The two possible GMM algorithms, corresponding to OMM and MM, are also asymptotically equivalent. We formulate this in the following theorem.

Theorem 5 Under Assumptions 1, 2, 3, 4, 8, 9, 10, 11, 12, 13 and 14 , and the condition

$L_{\mathbf{c}^{\dagger}}\left(\left\|J_{\mathbf{c}}\left(\mathbf{x}_{\mathbf{f}}^{*}\right)-S_{k}^{\dagger} J_{\mathbf{f}}\left(\mathbf{x}_{\mathbf{f}}^{*}\right)\right\|+\bar{M}\left\|\mathbf{f}\left(\mathbf{x}_{\mathbf{f}}^{*}\right)-\mathbf{y}\right\|\right)<1$ for $k \geq k_{0}$,

where $\bar{M}>0$ is a constant depending on the smoothness of the manifolds $\mathbf{f}(X)$ and $\mathbf{c}(X)$ but not on $k$, the GMM algorithms yield (linear) convergence to $\mathbf{x}_{\mathbf{f}}^{*}$.

Remark 14 In the case $S_{k+1}=J_{\mathbf{f}}\left(\mathbf{x}_{k+1}\right) J_{\mathbf{c}}^{\dagger}\left(\mathbf{x}_{k+1}\right)$, a lemma analogous to Lemma 5 can be trivially proved. In some situations the constant introduced in that lemma for GMM could be smaller than the one for MM. As a consequence, the (linear) convergence for the GMM schemes may be faster than for the MM iteration (see Sect. 5.1).

\section{Examples}

\subsection{Two simple examples}

First simple problem With this simple example we illustrate the convergence Theorems 4 and 5. The fine model is defined over $X=[-1,1]$ by

$\mathbf{f}(x)=\left[x, x^{2}\right]$.

The set $\mathbf{f}(X) \subset \mathbb{R}^{2}$ is part of a parabola and we want to find the point in that set closest in Euclidean norm to the specifications $\mathbf{y}=[3 / 4,0]$. The coarse model is defined over $Z=X$ and is the linear $\mathbf{c}(x)=[x,(1+x) / 2]$. Figure 5 shows a representation of the problem and the fine and coarse cost functions, $\|\mathbf{f}(x)-\mathbf{y}\|_{2}$ and $\|\mathbf{c}(x)-\mathbf{y}\|_{2}$, respectively. The corresponding optima are $x_{\mathbf{f}}^{*}=0.5$ and $x_{\mathbf{c}}^{*}=0.4$.
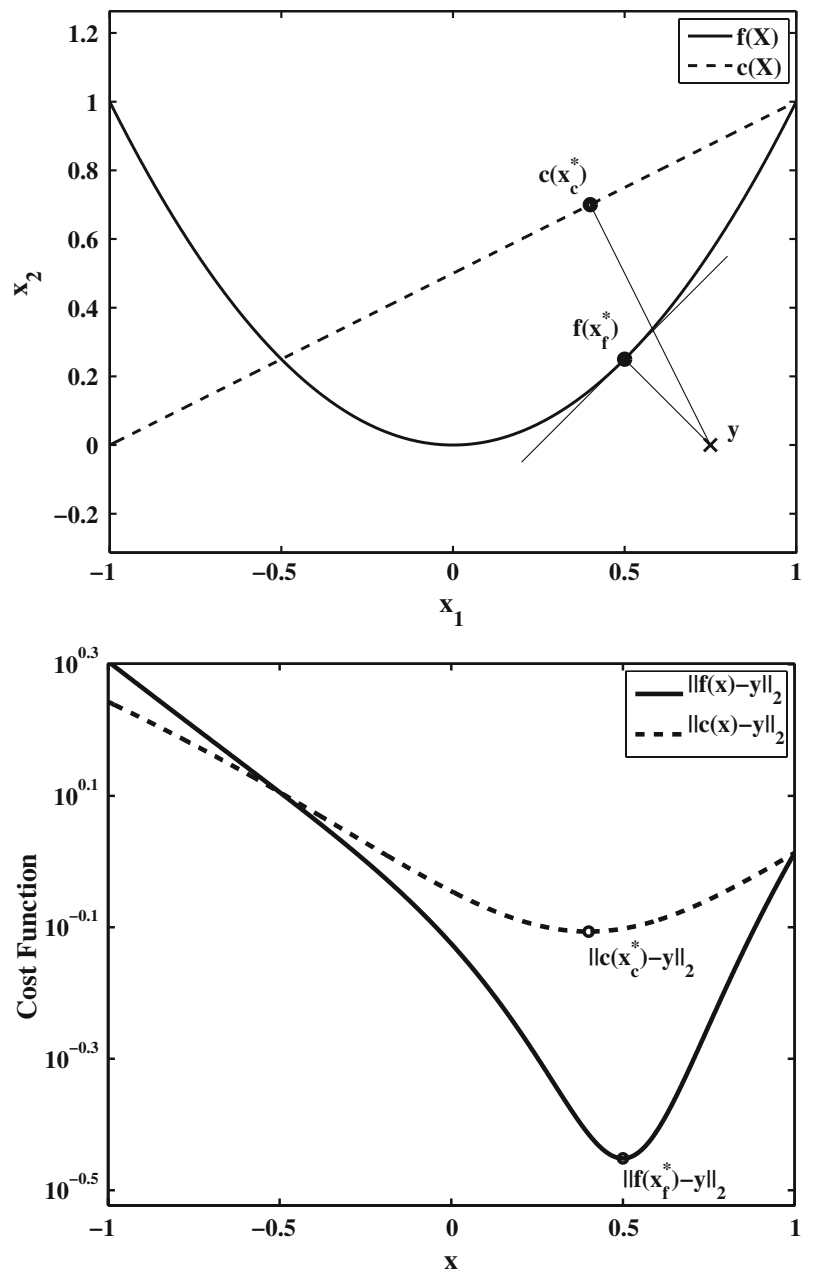

Fig. 5 Top the sets $\mathbf{f}(X)$ and $\mathbf{c}(X)$, specifications $\mathbf{y}$ and $\mathbf{f}\left(x_{\mathbf{f}}^{*}\right)$ and $\mathbf{c}\left(x_{\mathbf{c}}^{*}\right)$ for the first example. Bottom the fine and coarse cost functions, $\|\mathbf{f}(x)-\mathbf{y}\|_{2}$ and $\|\mathbf{c}(x)-\mathbf{y}\|_{2}$, respectively, for the same example

Though the two models are not specially similar around the solution region the similarity between them is sufficient for obtaining convergence with the manifold-mapping approach. Since both manifolds $\mathbf{f}(X)$ and $\mathbf{c}(X)$ are smooth, we expect a reasonable small constant $M$ in (50) for Theorem 4 . The design is not reachable and therefore convergence can be presumed to be linear for the MM and the GMM iterations (see Fig. 6). In this problem it is easy to check that both OMM and MM coincide, iterate by iterate. We see that MM needs 17 iterations for getting $\left|x_{k}-x_{\mathbf{f}}^{*}\right|$ smaller than $10^{-8}$. The GMM scheme, using the exact Jacobian, yields the same accuracy in 13 iterations. In Fig. 6 we also check that the constant in the linear convergence rate is smaller for the latter algorithm. If the Jacobian is estimated by Broyden's method, the complete iteration history coincides with that for MM. The reason is that for a function of one variable, Broyden's method coincides with the secant algorithm for approximating a derivative, and that procedure is essentially 


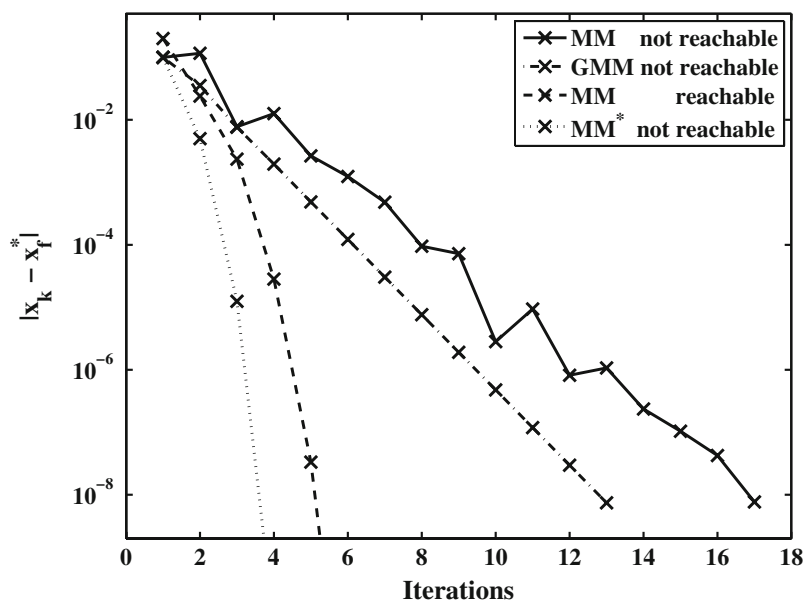

Fig. 6 Convergence history for $\left|x_{k}-x_{\mathbf{f}}^{*}\right|$ for the manifold-mapping $(M M)$ and generalized manifold-mapping $(G M M)$ iterations for reachable and non-reachable designs in the first simple example. MM $^{*}$ denotes the MM algorithm with $S_{k}=\bar{S}$

the one followed in the computation of $\Delta F$ and $\Delta C$ in the MM algorithm (not only MM and GMM use the same Jacobian estimation, also the iterates $x_{0}$ and $x_{1}$ coincide). In the next example, when we consider a function of two variables, we see that the Broyden-based GMM algorithm differs significantly from the MM algorithm.

In Fig. 6 we also observe two cases of superlinear convergence for MM (cf. Corollaries 3 and 4). If we apply MM with $S_{k}=\bar{S}$ (denoted by $\mathrm{MM}^{*}$ in Fig. 6) we obtain a solution with the same accuracy of $10^{-8}$ in only four iterations. Nevertheless that situation is unrealistic because the necessary information is not available before the optimization problem has been solved. In the case of the reachable design, given by $\mathbf{y}=[1 / 2,1 / 4]$ (yielding again $x_{\mathbf{f}}^{*}=0.5$ ), the solution is obtained with an accuracy of $10^{-8}$ in six iterations. The same superlinear convergence is observed for the GMM algorithm.

Second simple problem By means of this example with two design variables we show that different choices for the fine model Jacobian estimation at the $k$ th iteration, $\widetilde{J}_{\mathbf{f}}\left(\mathbf{x}_{k}\right)$, yield distinct convergence histories: the better $\widetilde{J}_{\mathbf{f}}\left(\mathbf{x}_{k}\right)$ approximates $J_{\mathbf{f}}\left(\mathbf{x}_{k}\right)$, the smaller the constant associated with the linear convergence. The example was introduced in [20] as a least squares best approximation of the data vector $\mathbf{y}=[0,-0.4$, 0.1 by the fine model

$\mathbf{f}(\mathbf{x})=\mathbf{f}\left(x_{1}, x_{2}\right)=\left[x_{1}\left(x_{2}-1\right)^{2}, x_{1}, x_{1}\left(x_{2}+1\right)^{2}\right]$

defined over $X=\mathbb{R}^{2}$. It can be seen that the design is not reachable. The coarse model

$\mathbf{c}(\mathbf{x})=\mathbf{c}\left(x_{1}, x_{2}\right)=\left[-x_{1}+x_{2}, x_{2}, x_{1}+x_{2}\right]$

is again linear and it is also defined over $Z=X$.

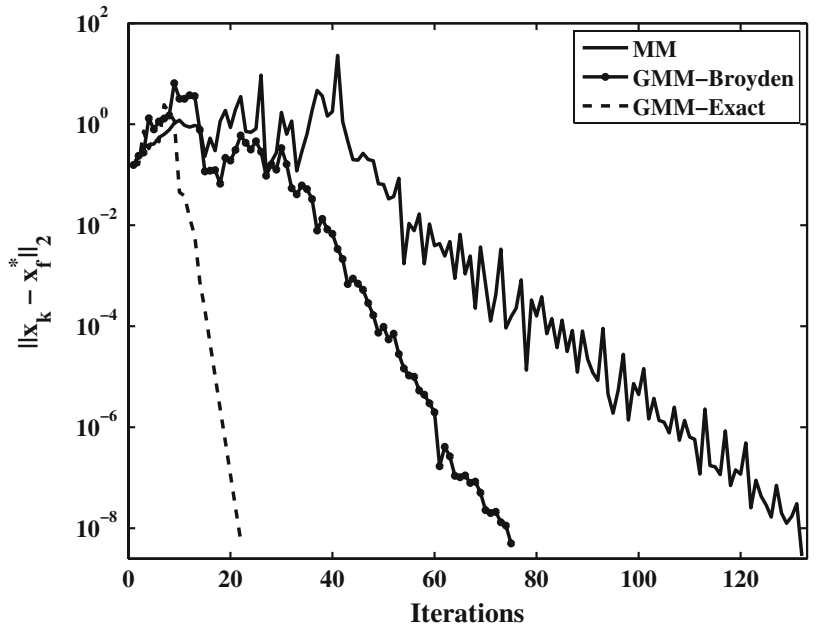

Fig. 7 Convergence history for $\left\|\mathbf{x}_{k}-\mathbf{x}_{\mathbf{f}}^{*}\right\|$ for the manifold-mapping $(M M)$ and two different generalized manifold-mapping (GMM) iterations in the second simple example (non-reachable design and a bad correspondence between the fine and the coarse model). GMMExact denotes the GMM scheme with the exact Jacobian $J_{\mathbf{f}}\left(\mathbf{x}_{k}\right)$. GMM-Broyden approximates that Jacobian via Broyden's method

We solve the problem with the MM and the GMM algorithms. For GMM, two variants are compared, one with the exact Jacobian for the fine model and the other with an approximation based on Broyden's method. It should be noticed that for most time-expensive fine models, the availability of the exact Jacobian is an unrealistic assumption. All the schemes yield the fine optimum $\mathbf{x}_{\mathbf{f}}^{*}=[-0.101,-0.141]$. The convergence history for the three methods is shown in Fig. 7. We clearly observe that the convergence is linear in all cases and that the constant $M$ in the convergence theorems (mean slope of the convergence history) is different for each algorithm. In this problem the discrepancy between fine and coarse models in the solution region is large and this fact is recognized in a large number of iterations compared with the previous example. In practice, fine and coarse models are much more similar and, hence, convergence is generally achieved in significantly fewer iterations.

\subsection{Practical examples}

The next two examples are design problems from practice $[21,22]$. We show that they can be solved efficiently by the manifold-mapping approach. Since-in both cases-Jacobians for the fine model are not available, Broyden's method is used to estimate $J_{\mathbf{f}}\left(\mathbf{x}_{k}\right)$ when the GMM iteration is used. In the two optimal design problems the inequality constraints $\overline{\mathbf{k}}(\mathbf{x})$ are easy to compute (box constraints).

\subsubsection{Coreless actuator [21]}

A magnetic actuator is a device that converts magnetic energy into mechanical force and motion. The one in Fig. 8 (left) 


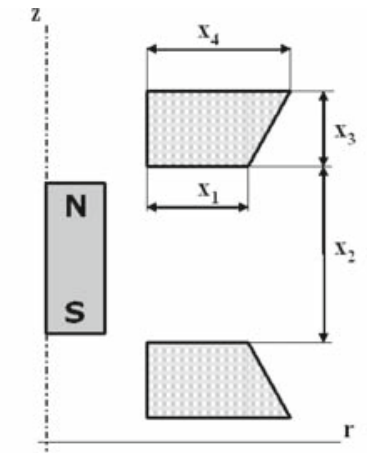

MAGNET

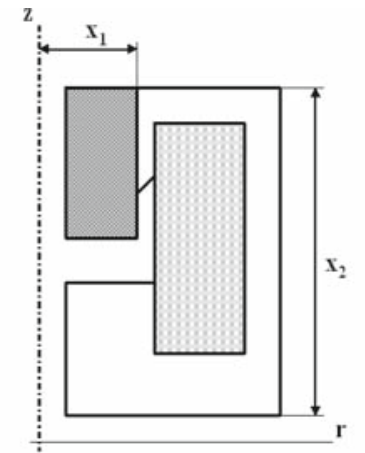

CORE

\section{PLUNGER}

Fig. 8 Geometries of the coreless (left) and automotive (right) actuators. The axis of rotational symmetry is denoted by $\mathbf{z}$

consists of a moving cylindrical magnet and two fixed toroidal coils. It is called coreless because actuators usually have ferromagnetic cores. The specification $\mathbf{y}$ is a force response exerted on the magnet. This is specified as the force at a finite number of displacements of the magnet along the symmetry axis. The actuator geometry $\mathbf{x}=\left[x_{1}, x_{2}, x_{3}, x_{4}\right]$ (see Fig. 8) has to be determined so that the discrepancy in the Euclidean norm between the computed and specified force response is minimized. The fine model is based on a calculation of the forces after a finite-element computation of the vector magnetic potential formulation [34] of the axisymmetric magnetostatic equations. In the coarse model, the force is computed for a much simplified model, where the actuator is represented by only a (small) number of coils. Depending on the number of coils in the simplification, several coarse models are obtained. The coarse model computation used here is around 300 times faster than the fine model one. More details on this particular problem can be found in [21].

The coreless actuator optimization results are shown in Table 1. Both the manifold and the Broyden-based GMM algorithms yield an acceptable design solution after only two fine model evaluations. The number of coarse model evaluations is larger for the second scheme because the coarse model Jacobian is approximated by finite differences in each iteration step. In an earlier paper [21] the space-mapping technique was reported to be a very efficient solver for this problem. In order for space mapping to obtain a value for the cost function comparable with one found by the two manifold-mapping algorithms in Table 1, all coarse models from [21] are considered. The best space-mapping option found is used in our comparison.

\section{Automotive actuator [22]}

Automotive actuators are used in devices such as electromagnetic switches, relays, valves, etc. and they typically generate high levels of force. The one in Fig. 8 (right) consists of a core and a plunger, both made of iron, and a copper coil. The design variables are the sizes $\mathbf{x}=\left[x_{1}, x_{2}\right]$ indicated in the figure. Again, the specification $\mathbf{y}$ is a force response exerted over the plunger, when it moves vertically. The Euclidean norm of the difference between the computed force for a given $\mathbf{x}$ and the specification $\mathbf{y}$ is minimized, keeping the device volume constant. (Elimination of this easyto-compute equality constraint leads to the two mentioned design variables.) A finite element computation for the axisymmetric magnetostatic equations plus a post-processing stage make the fine model. The coarse model is based on an equivalent magnetic circuit [16] of the actuator (analogous to an electrical circuit) and it is around 400 times faster than the fine one. It should be noticed that the fine model optimum $\mathbf{x}_{\mathbf{f}}^{*}$ lies on the boundary of the set $X$. Additional problem details can be found in [22].

Table 2 shows the results for the automotive actuator optimization. The cost function corresponding to the solutions has a considerably larger value than in the previous example because the specification $\mathbf{y}$ has a significantly larger norm. (This cost function is not a relative measure of the force discrepancy.) When these facts are taken into account, the optimal cost function values are similar for both design problems. The manifold-mapping algorithm yields the solution after three fine model function evaluations. GMM, with the fine model Jacobian estimated via Broyden's method, performs almost identically. (The coarse model Jacobian computations are recognized in a small increase in the coarse

Table 1 Optimizing the coreless actuator

\begin{tabular}{lll}
\hline & $\#(f, c)$ evals & Cost function \\
\hline MM & $(2,132)$ & 2.2 \\
GMM & $(2,148)$ & 2.2 \\
SM & $(3,150)$ & 2.3 \\
\hline
\end{tabular}

$M M$ manifold mapping, GMM generalized manifold mapping, $S M$ space mapping

The second column shows the total amount of fine and coarse model evaluations needed in the optimization. The coarse model used is approximately 300 times faster than the fine one

Table 2 Optimizing the automotive actuator

\begin{tabular}{lll}
\hline & $\#(f, c)$ evals. & Cost function \\
\hline MM & $(3,66)$ & 45.0 \\
GMM & $(3,78)$ & 45.0 \\
SM & $(6,296)$ & 45.3 \\
SQP & $(12,47)$ & 45.0 \\
\hline
\end{tabular}

$M M$ manifold mapping, GMM generalized manifold mapping, $S M$ space mapping, $S Q P$ sequential quadratic programming

The second column shows the total amount of fine and coarse model evaluations needed in the optimization. The coarse model used is approximately 400 times faster than the fine one. SQP takes the coarse model optimum $\mathbf{x}_{\mathbf{c}}^{*}$ as its initial guess 
model function evaluations for GMM algorithm.) The usual (standard) optimization method for these problems, sequential quadratic programming (SQP) [29], is applied to the fine model cost function with the coarse model optimum $\mathbf{x}_{\mathbf{c}}^{*}$ as initial guess. A design with a cost function value comparable with the one obtained with the manifold-mapping approach is obtained with almost four times the computational cost. Space mapping, on the other side, performs faster than sequential quadratic programming but it is less efficient than manifold mapping. In this case we also observe the wellknow fact that standard SM does not converge to the accurate solution [20].

\section{Conclusion}

Manifold mapping was introduced in [20] as an efficient twolevel approach for optimization, to be considered for very time-demanding design problems. In this paper a number of possible variants are identified: OMM, MM and GMM. $\mathrm{OMM}$ and $\mathrm{MM}$ are asymptotically equivalent and they perform identically in most practical situations. MM is preferred to OMM because it is easier to implement (see also [27]). GMM represents a whole family of schemes based on the manifold-mapping approach. If a better approximation for $J_{\mathbf{f}}\left(\mathbf{x}_{k}\right)$ than $\Delta F \Delta X_{k}^{-1}$ is available, then GMM is recommended. In all cases, if the iteration converges, the solution of the accurate model is the fixed point of the iteration. Conditions for convergence have been given for all algorithms described. By two simple examples we illustrate some of the theoretical aspects dealt with in the paper. The practical performance of the presented algorithms is illustrated by two cases of optimal design in electromagnetics.

Acknowledgments We thank W. Hoffmann and D. Lahaye for their fruitful discussions during the development of this work.

\section{Appendix}

Definitions for differentiable manifolds

Because the concept of manifold is central in our description of the optimization methods studied in this paper, in this appendix we summarize a number of basic notions, from [36], related with manifolds.

Definition 1 An $n$-dimensional differentiable manifold of class $C^{k}$ with $1 \leq k \leq \infty$ is a pair $(M, \mathcal{F})$ consisting of an $n$-dimensional, second countable, locally Euclidean space $M$ together with a differentiable structure of class $C^{k}$. Usually, the differentiable manifold $(M, \mathcal{F})$ is denoted as $M$.

Definition 2 A locally Euclidean space $M$ of dimension $n$ is a Hausdorff topological space $M$ for which each point has a neighborhood homeomorphic to an open subset of the Euclidean space $\mathbb{R}^{n}$.

Definition 3 A differentiable structure $\mathcal{F}$ of class $C^{k}(1 \leq$ $k \leq \infty)$ on a locally Euclidean space $M$ is a collection of coordinate systems (also called an atlas of charts) $\left\{\left(U_{\alpha}, \varphi_{\alpha}\right)\right.$ : $\alpha \in A\}$ satisfying:

(a) $\bigcup_{\alpha \in A} U_{\alpha}=M$,

(b) $\varphi_{\alpha} \circ \varphi_{\beta}^{-1}$ is $C^{k}$ for every $\alpha, \beta \in A$,

(c) the collection $\mathcal{F}$ is maximal with respect to (b); that is, if $(U, \varphi)$ is a coordinate system such that $\varphi \circ \varphi_{\alpha}^{-1}$ and $\varphi_{\alpha} \circ \varphi^{-1}$ are $C^{k}$-functions for all $\alpha \in A$, then $(U, \varphi) \in \mathcal{F}$.

\section{References}

1. Alexandrov, N., Dennis, J.E. Jr.., Lewis, R.M., Torczon, V.: A trust region framework for managing the use of approximation models in optimization. Struct. Multidiscip. Optim. 15, 16-23 (1998)

2. Bakr, M.H., Bandler, J.W., Biernacki, R.M., Chen, S.H., Madsen, K.: A trust region aggressive space mapping algorithm for EM optimazation. IEEE Trans. Microw. Theory Tech. 46, 24122425 (1998)

3. Bakr, M.H., Bandler, J.W., Ismail, M.A., Rayas-Sanchez, J.E., Zhang, Q.J.: Neural space-mapping optimization for EM-based design. IEEE Trans. Microw. Theory Tech. 48, 2307-2315 (2000)

4. Bandler, J.W., Biernacki, R.M., Chen, S.H., Grobelny, P.A., Hemmers, R.H.: Space mapping technique for electromagnetic optimization. IEEE Trans. Microw. Theory Tech. 42, 25362544 (1994)

5. Bandler, J.W., Biernacki, R.M., Chen, S.H., Hemmers, R.H., Madsen, K.: Electromagnetic optimization exploiting aggressive space mapping. IEEE Trans. Microw. Theory Tech. 43, 28742882 (1995)

6. Bandler, J.W., Cheng, Q.S., Dakroury, A.S., Mohamed, A.S., Bakr, M.H., Madsen, K., Sondergaard, J.: Space mapping: the state of the art. IEEE Trans. Microw. Theory Tech. 52, 337-361 (2004)

7. Bandler, J.W., Cheng, Q.S., Nikolova, N.K., Ismail, M.A.: Implicit space mapping optimization exploiting preassigned parameters. IEEE Trans. Microw. Theory Tech. 52, 378-385 (2004)

8. Bandler, J.W., Hailu, D.M., Madsen, K., Pedersen, F.: A spacemapping interpolating surrogate algorithm for highly optimized EM-based design of microwave devices. IEEE Trans. Microw. Theory Tech. 52(11), 2593-2600 (2004)

9. Barthelemy, J.F., Haftka, R.: Approximation concepts for optimum structural design - a review. Struct. Optim. 5, 129-144 (1993)

10. Böhmer, K., Hemker, P.W., Stetter, H.J.: The defect correction approach. In: Böhmer, K., Stetter, H.J. (eds.) Defect Correction Methods: Theory and Applications, Computing Suppl. 5, pp. 1-32. Springer, Berlin (1984)

11. Böhmer, K., Stetter, H.J.: Defect Correction Methods: Theory and Applications. Springer, Berlin (1984)

12. Booker, A.J., Dennis, J.E. Jr., Frank, P.D., Serafini, D.B., Torczon, V., Trosset, M.W.: A rigorous framework for optimization of expensive functions by surrogates. Struct. Multidiscip. Optim. 17, 1-13 (1999)

13. Braibant, V., Fleury, C.: An approximation-concepts approach to shape-optimal design. Comput. Methods Appl. Mech. Eng. 53, 119-148 (1985)

14. Broyden, C.G.: A class of methods for solving nonlinear simultaneous equations. Math. Comput. 19, 577-593 (1965) 
15. Buhmann, M.D., Ablowitz, M.J.: Radial Basis Functions: Theory and Implementations. Cambridge University, London (2003). ISBN 0-521-63338-9

16. Cheng, D.K.: Field and Wave Electromagnetics. Addison-Wesley (1989)

17. DeBoor, C., Ron, A.: Computational aspects of polynomial interpolation in several variables. Math. Comput. 58, 705-727 (1992)

18. Dennis, J.E. Jr., Torczon, V.: Managing approximation models in optimization. In: Alexandrov, N.M., Hussaini, M.Y. (eds.) Multidisciplinary Design Optimization: State-of-the-Art, pp. 330-347. SIAM, Philadelphia (1997)

19. Echeverría, D.: Two new variants of the manifold-mapping technique. COMPEL Int. J. Comput. Math. Electr. Electron. Eng. 26(2), 334-344 (2007)

20. Echeverría, D., Hemker, P.W.: Space mapping and defect correction. Comput. Methods Appl. Math. 5(2), 107-136 (2005)

21. Echeverría, D., Lahaye, D., Encica, L., Hemker, P.W.: Optimisation in electromagnetics with the space-mapping technique. COMPEL Int. J. Comput. Math. Electr. Electron. Eng. 24(3), 952-966 (2005)

22. Echeverría, D., Lahaye, D., Encica, L., Lomonova, E.A., Hemker, P.W., Vandenput, A.J.A.: Manifold-mapping optimization applied to linear actuator design. IEEE Trans. Magn. 42(4), 1183-1186 (2006)

23. Giunta, A.A., Eldred, M.S.: Implementation of a trust region model management strategy in the DAKOTA optimization toolkit. In: Proceedings of the 8th AIAA/USAF/NASA/ISSMO Symposium on Multidisciplinary Analysis and Optimization. AIAA (2000). Paper AIAA-2000-4935

24. Golub, G.H., VanLoan, C.F.: Matrix Computations. North Oxford Academic, Oxford (1983)
25. Griewank, A.: Evaluating derivatives. Principles and techniques of algorithmic differentiation. SIAM Frontiers in Applied Mathematics, vol. 19. SIAM, Philadelphia (2000)

26. Hackbusch, W.: Multigrid methods and applications. Series in Computational Mathematics, vol. 4. Springer, Berlin (1985)

27. Hemker, P.W., Echeverría, D.: A trust-region strategy for manifoldmapping optimization. J. Comput. Phys. 224, 464-475 (2007)

28. Journel, A.G., Huijbregts, C.J.: Mining Geostatistics. Academic Press, London (1981)

29. Nocedal, J., Wright, S.J.: Numerical optimization. Springer Series in Operations Research. Springer, Heidelberg (1999)

30. Owen, A.B.: Orthogonal arrays for computer experiments, integration and visualization. Stat. Sin. 2, 439-452 (1992)

31. Pedersen, F., Weitzmann, P., Svendsen, S.: Modeling thermally active building components using space mapping. In: Proceedings of the 7th Symposium on Building Physics in the Nordic Countries, pp. 896-903 (2005). ISBN: 9979-9174-6-6

32. Pironneau, O.: Optimal Shape Design For Elliptic Systems. Springer, Heidelberg (1984)

33. Schmit, L.A. Jr., Farshi, B.: Some approximation concepts for structural synthesis. AIAA J. 12, 692-699 (1974)

34. Silvester, P.P., Ferrari, R.L.: Finite Elements for Electrical Engineers. Cambridge University Press, New York (1996)

35. Stein, M.: Large sample properties of simulating using latin hypercube sampling. Technometrics 29(2), 143-151 (1987)

36. Warner, F.W.: Foundations of differentiable manifolds and lie groups. Graduate Texts in Mathematics. Springer, Heidelberg (1983). ISBN 0-387-90894-3 